\title{
Computed tomographic evaluation of myocardial ischemia
}

\author{
Yuki Tanabe ${ }^{1}$ - Akira Kurata ${ }^{1} \cdot$ Takuya Matsuda $^{1} \cdot$ Kazuki Yoshida $^{1} \cdot$ Dhiraj Baruah $^{2} \cdot$ Teruhito Kido $^{1}$. \\ Teruhito Mochizuki ${ }^{1,3} \cdot$ Prabhakar Rajiah $^{4}$
}

Received: 25 November 2019 / Accepted: 20 January 2020 / Published online: 5 February 2020

(c) The Author(s) 2020

\begin{abstract}
Myocardial ischemia is caused by a mismatch between myocardial oxygen consumption and oxygen delivery in coronary artery disease (CAD). Stratification and decision-making based on ischemia improves the prognosis in patients with CAD. Non-invasive tests used to evaluate myocardial ischemia include stress electrocardiography, echocardiography, single-photon emission computed tomography, and magnetic resonance imaging. Invasive fractional flow reserve is considered the reference standard for assessment of the hemodynamic significance of CAD. Computed tomography (CT) angiography has emerged as a first-line imaging modality for evaluation of $\mathrm{CAD}$, particularly in the population at low to intermediate risk, because of its high negative predictive value; however, CT angiography does not provide information on the hemodynamic significance of stenosis, which lowers its specificity. Emerging techniques, e.g., CT perfusion and CT-fractional flow reserve, help to address this limitation of CT, by determining the hemodynamic significance of coronary artery stenosis. CT perfusion involves acquisition during the first pass of contrast medium through the myocardium following pharmacological stress. CTfractional flow reserve uses computational fluid dynamics to model coronary flow, pressure, and resistance. In this article, we review these two functional CT techniques in the evaluation of myocardial ischemia, including their principles, technology, advantages, limitations, pitfalls, and the current evidence.
\end{abstract}

Keywords Computed tomography $\cdot$ Coronary artery disease $\cdot$ Myocardial ischemia $\cdot$ Myocardial perfusion $\cdot$ Fractional flow reserve

\section{Introduction}

Coronary artery atherosclerosis progresses asymptomatically in the early stage and leads to luminal stenosis and myocardial ischemia [1]. The ischemic cascade illustrates

Electronic supplementary material The online version of this article (https://doi.org/10.1007/s11604-020-00922-8) contains supplementary material, which is available to authorized users.

Teruhito Kido

t.k.ehime@gmail.com

1 Department of Radiology, Ehime University Graduate School of Medicine, Shitsukawa, Toon, Ehime 791-0295, Japan

2 Department of Radiology, Medical College of Wisconsin, Milwaukee, WI, USA

3 Department of Radiology, I.M. Sechenov First Moscow State Medical University, Bol'shaya Pirogovskaya Ulitsa, Moscow, Russia

4 Department of Radiology, Mayo Clinic, Rochester, MN, USA the progressive pathological conditions that develop from hemodynamically significant stenosis, evolving from subclinical to clinical stages (Fig. 1) [2-4]. Decreased perfusion leads to metabolic changes, followed by diastolic and then systolic dysfunction, electrocardiographic (ECG) changes, and anginal chest pain [3]. Blood flow and contractile function in myocardial ischemia can be improved by medical therapy or revascularization procedures such as percutaneous coronary intervention (PCI) or coronary artery bypass grafting (CABG). Large multicenter trials have demonstrated improvement in the prognosis of coronary artery disease (CAD) by decision-making according to myocardial ischemia shown on stress testing $[5,6]$. Therefore, current international guidelines require proof of myocardial ischemia before a revascularization procedure $[7,8]$. This can be achieved by multiple noninvasive and invasive tests, each with advantages and disadvantages (Table 1).

Noninvasive tests include exercise ECG, echocardiography, nuclear imaging, stress magnetic resonance imaging (MRI), and computed tomography (CT) and evaluate 


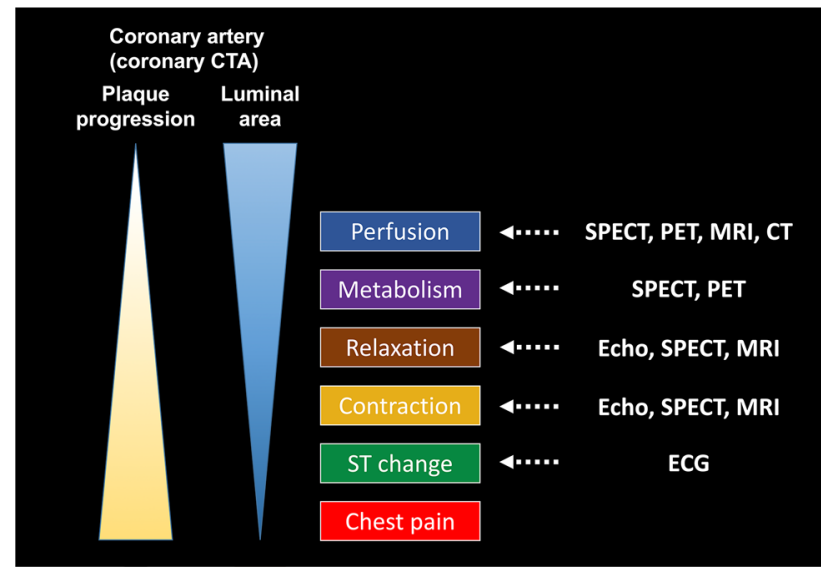

Fig. 1 Illustration showing the progressive pathological conditions in the myocardial ischemic cascade. Coronary artery atherosclerosis progresses and leads to myocardial hypoperfusion because of plaque progression and luminal stenosis. Myocardial hypoperfusion is followed by metabolic abnormalities, diastolic dysfunction, systolic dysfunction, and ECG abnormalities, culminating in chest pain. The right column shows the modalities that can be used to detect abnormalities at each step of the cascade. $C T$ computed tomography, CTA computed tomography angiography, ECG electrocardiogram, SPECT single-photon emission computed tomography, PET positron emission tomography, $M R I$ magnetic resonance imaging

different stages along the ischemic cascade. The choice of test to symptomatic patients is determined by the pretest probability of CAD and the inspection characteristics including invasiveness, cost, and accessibility. Cardiac CT is widely used as coronary CT angiography (CTA), allowing direct visualization of coronary artery stenosis, and plays an important role in the diagnostic management of CAD [7]. The other non-invasive tests can evaluate myocardial ischemia, but leave some difficulties on the detection of culprit lesion causing ischemia. The invasive fractional flow reserve (FFR) can evaluate the lesion-specific ischemia compared to the morphological stenosis assessed by invasive coronary angiography (ICA) under the conditions of maximum hyperemia $[9,10]$. The FFR correlates poorly with stenosis on ICA, and many stenoses that are significant on ICA $(\geq 50 \%$ stenosis) do not have a significant low FFR $(\leq 0.8)$ $[5,10]$. The clinical outcome has been shown to be better when revascularization decisions are based on the FFR than on visual estimation of the severity on ICA [5, 11-13].

Japan has the most computed tomography (CT) scanners among those of the Organization for Economic Co-operation and Development (OECD) countries [14]. In Japan, cardiac $\mathrm{CT}$ is more common than other modalities (MRI, SPECT, PET), and the number of examinations is also increasing because of the high accessibility and diagnostic performance for detecting coronary artery stenosis [15]. Recent CT technological developments such as high-speed gantry rotation, wide-detector, and iterative reconstruction have increased the value of coronary CTA. Moreover, they allow us to assess myocardial ischemia using stress CTP or CT-derived FFR estimation based on computational flow dynamics. In this review, we review the clinical usefulness of CT-based diagnostic tools in assessing myocardial ischemia from the methodology to advantages and clinical limitations.

\section{Coronary CTA in CAD}

Coronary CTA has high diagnostic performance for prediction of significant coronary stenosis on ICA (i.e., $\geq 50 \%$ ) with sensitivity of $89 \%$, specificity of $96 \%$, positive predictive value (PPV) of $78 \%$, and negative predictive value (NPV) of $98 \%$ on per-segment basis [16]. The high NPV makes coronary CTA valuable for excluding CAD in the population at low or intermediate risk and acting as a gatekeeper for ICA. Up to $63 \%$ of elective ICA procedures show non-obstructive disease despite previous functional tests, resulting in suboptimal resource utilization [17]. However, CTA is limited in terms of revealing the hemodynamic significance of a stenosis, given that there is no correlation between the severity of stenosis on CTA and the functional consequences. Only $49 \%$ of significant coronary stenosis on CTA ( $\geq 50 \%$ reduction in diameter) correlates with the gold standard of invasive FFR $(<0.75)$ [18]. Possible causes for this poor correlation include visual overestimation of luminal stenosis and classification of lesions with heavy calcification or motion as "positive" [19]. As a result of the poor specificity, patients with $\geq 50 \%$ stenosis on CTA are recommended to undergo further investigation with a functional test to estimate the hemodynamic significance of the stenosis [20].

\section{CT perfusion}

\section{Principle of CTP}

CTP evaluates the first pass of contrast medium through the myocardium at rest and during pharmacological stress. CTP emphasizes the differences in perfusion between normal and ischemic myocardium (Fig. 2) [21]. Myocardial perfusion is a complex process involving the coronary arteries and microvasculature. Coronary artery stenosis decreases the myocardial blood flow and perfusion pressure whereas the microvessels dilate to decrease resistance and maintain resting myocardial perfusion even at $80 \%$ luminal stenosis [22]. Autoregulation is limited at rest when the stenosis is severe and during stress even in the earlier stages due to higher myocardial oxygen consumption in these states [22]. 
Table 1 Advantages and disadvantages of different tests used in the evaluation of myocardial ischemia

\begin{tabular}{|c|c|c|}
\hline Modality & Advantages & Disadvantages \\
\hline \multirow[t]{3}{*}{ Electrocardiogram } & Availability & Depends on patient activity and cooperation with the test \\
\hline & Cost-effectiveness & Low sensitivity for ischemia \\
\hline & $\begin{array}{l}\text { Multipurpose use for ischemia, exercise tolerance, and } \\
\text { therapeutic effect }\end{array}$ & Diagnostic difficulty on a per-vessel basis \\
\hline \multirow[t]{3}{*}{ Echocardiogram } & High temporal resolution & Two-dimensional cross-sectional images \\
\hline & Differential assessment of diastolic and systolic dysfunction & $\begin{array}{l}\text { Depends on operator experience, image quality, and limited } \\
\text { acoustic window }\end{array}$ \\
\hline & Myocardial strain imaging & \\
\hline \multirow[t]{5}{*}{$\mathrm{CT}$} & High spatial resolution & Radiation exposure \\
\hline & Assessment of coronary artery stenosis and plaque & Limited temporal resolution \\
\hline & CT perfusion (ischemia) & Intolerance to irregular heartbeat \\
\hline & Late iodine enhancement (infarction) & Contrast contamination by preceding protocol \\
\hline & $\begin{array}{l}\text { CT-FFR (computational lesion-specific assessment of myo- } \\
\text { cardial ischemia) }\end{array}$ & $\begin{array}{l}\text { Contrast-related complications (kidney, allergy, and chronic } \\
\text { lung disease) }\end{array}$ \\
\hline \multirow{5}{*}{$\begin{array}{r}\text { Nuclear imaging } \\
\text { (SPECT, PET) }\end{array}$} & Abundant evidence & Less spatial resolution \\
\hline & Tracer selection by purpose & No information on coronary anatomy \\
\hline & ECG-gated scan (perfusion and wall motion) & Radiation dose \\
\hline & Image fusion & Cost and throughput \\
\hline & Myocardial viability & \\
\hline \multirow[t]{4}{*}{ MRI } & High spatial resolution & Contraindications (metallic device, claustrophobia) \\
\hline & High contrast resolution & $\begin{array}{l}\text { Contrast-related complications (brain deposition, nephrogenic } \\
\text { systemic fibrosis) }\end{array}$ \\
\hline & No ionizing radiation exposure & Throughput (long examination time) \\
\hline & Differentiation of ischemia and infarction & Susceptible to arrhythmia \\
\hline \multirow[t]{2}{*}{ Invasive FFR } & Lesion-specific assessment of myocardial ischemia & Invasive procedure and risk of complications \\
\hline & Established evidence for decision-making and prognosis & Complexity for repeat pharmacological stress \\
\hline
\end{tabular}

$C T$ computed tomography, ECG electrocardiogram, FFR fractional flow reserve, $M R I$ magnetic resonance imaging, PET positron emission tomography, SPECT single-photon emission computed tomography

\section{Stress agents for CTP}

Hyperemia is induced by intravenous administration of a vasodilator stress agent, such as adenosine, adenosine triphosphate (ATP), dipyridamole, or regadenoson. Adenosine binds to the A2A adenosine receptor, leading to coronary vasodilation. It is injected at a rate of $0.14 \mathrm{mg} / \mathrm{kg} /$ min for 6 min and has a rapid onset of action and a short half-life of 1-5 s. ATP is metabolized to adenosine and acts in the same way as adenosine. It is injected intravenously at a rate of $0.16 \mathrm{mg} / \mathrm{kg} / \mathrm{min}$ for $5 \mathrm{~min}$ and has a half-life of $\leq 20 \mathrm{~s}$. Both these agents are cleared by cellular mechanisms. Dipyridamole acts indirectly by preventing intracellular reuptake and transport of adenosine by endothelial cells and by increasing endogenous adenosine levels. It is injected intravenously at a rate of $0.14 \mathrm{mg} / \mathrm{kg} / \mathrm{min}$ for $4 \mathrm{~min}$, has a half-life of 30-45 min, and is cleared by liver. Adenosine, ATP, and dipyridamole are non-selective adenosine receptor agonists and cause flushing, headache, lightheadedness, and chest discomfort, and these side effects are naturally treated due to the very short half-life of adenosine or ATP. Regarding dipyridamole, aminophylline (50-250 mg intravenously at least $1 \mathrm{~min}$ after the tracer injection) is used if necessary. Regadenoson is a selective A2A agonist that has a lower risk of side effects. It is injected as a bolus of $0.4 \mathrm{mg}$ over $10 \mathrm{~s}$ and is followed by a saline flush; it has a half-life of 33-108 s and is cleared by the kidney [23-25]. Patients should be instructed to avoid consuming caffeine-containing products for $\geq 24 \mathrm{~h}$ prior to the scheduled test to prevent the interference with the coronary vasodilatory effects.

\section{Acquisition techniques of CTP}

\section{Static CTP imaging}

Static CTP refers to the single sampling of perfusion during the first pass of iodinated contrast in the myocardium (Fig. 3a) [26]. Scan timing is critical for detecting the perfusion abnormality when this technique is used. A bolus-tracking or timing-bolus technique can aid accurate calculation 
Fig. 2 Time attenuation curves for normal myocardium (blue) and ischemic myocardium (orange). At the optimal scan time (B), stress CT perfusion demonstrates a clear distinction between a normal and ischemic myocardium because of large differences in attenuation between normal and abnormal myocardium. Stress CT perfusion cannot distinguish normal from ischemic myocardium if the timing is too early (A) or too late (C). $C T$ computed tomography, $H U$ Hounsfield unit

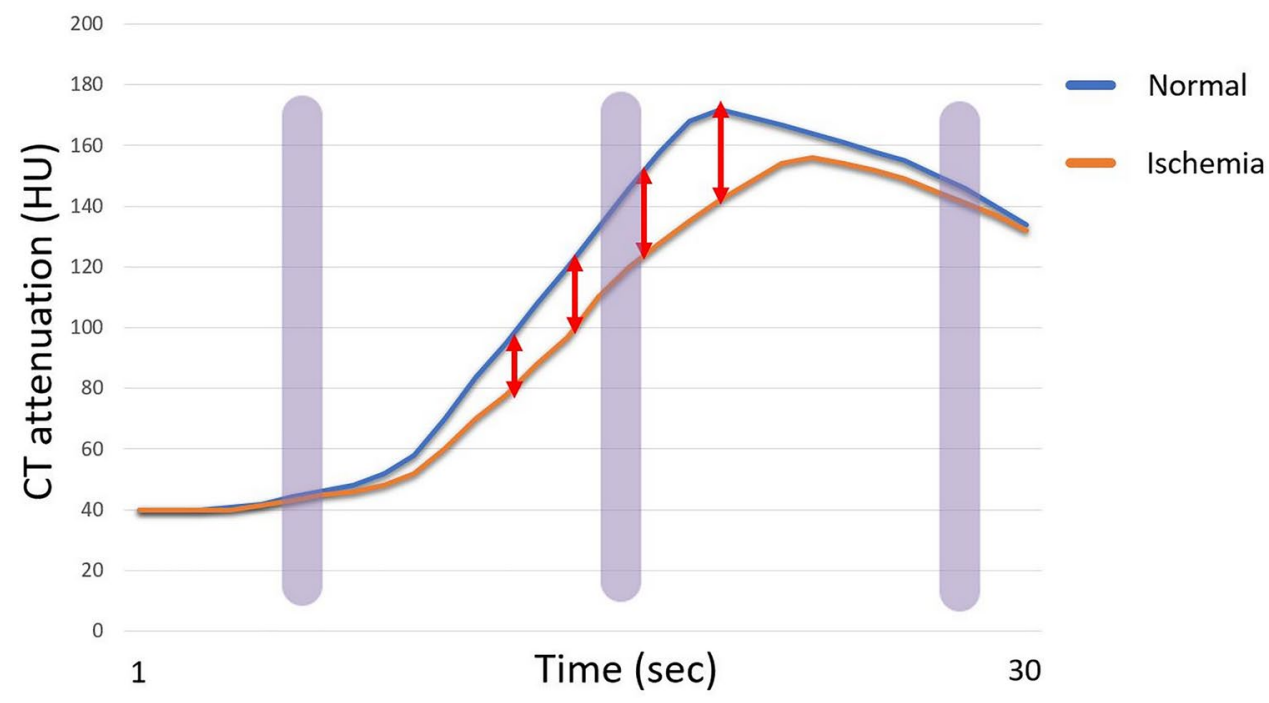

A

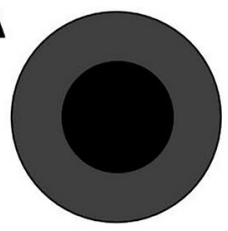

B

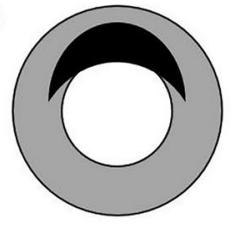

C

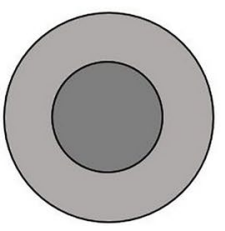

\section{Static CTP}

A

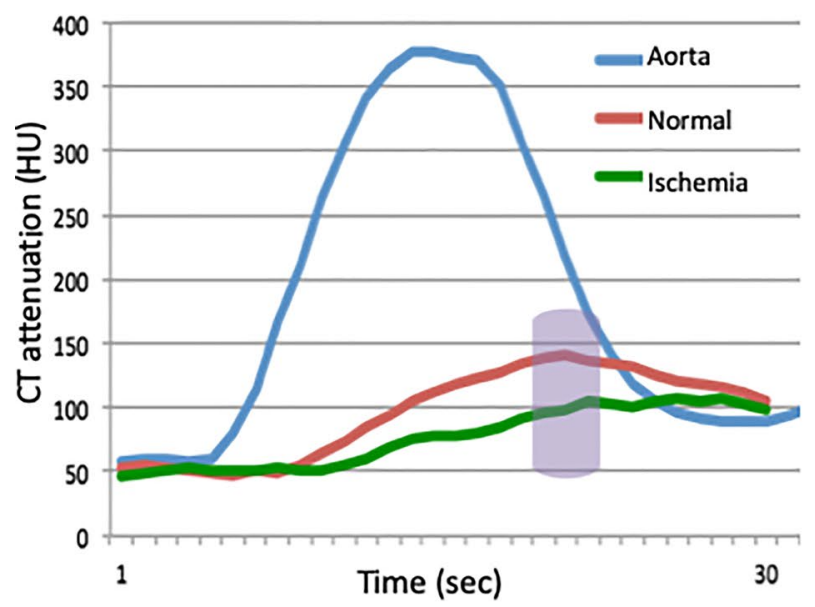

Single scan

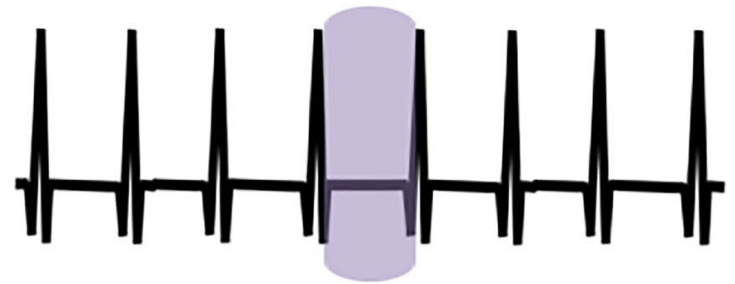

Fig. 3 Static and dynamic CTP techniques. a Static CTP data are acquired during a single phase of first pass of contrast in the myocardium. b Dynamic CTP data are acquired at multiple phases of first

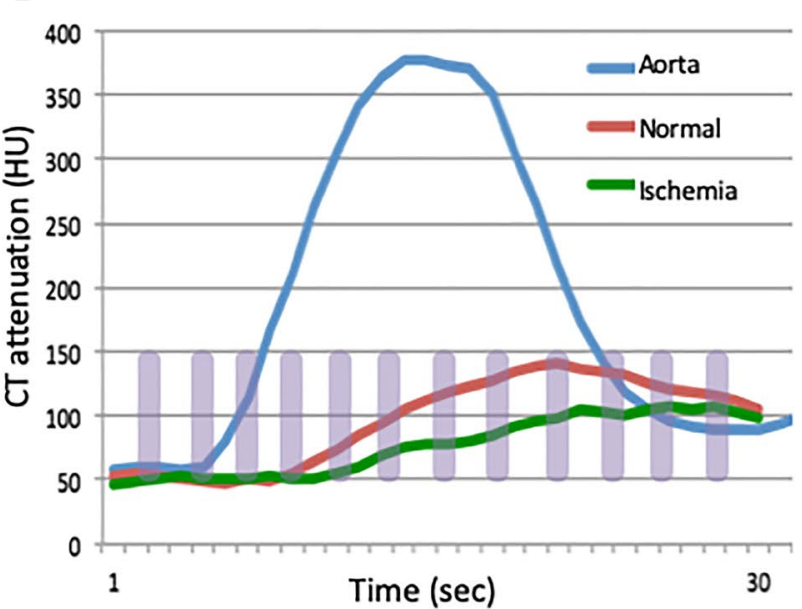

Multiple scan

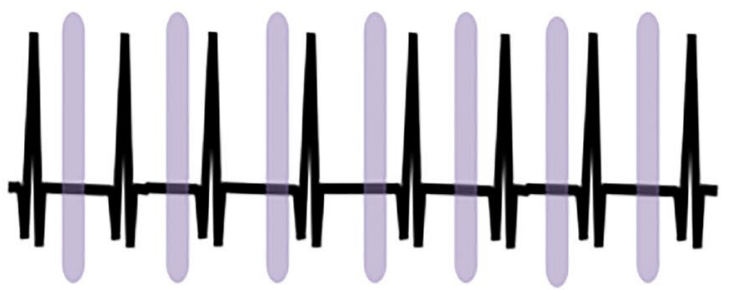

pass of contrast in the myocardium. Blue box means scan duration. $C T P$ computed tomography perfusion, $H U$ Hounsfield unit 
of the scan time. The optimal scan time is approximately $2-10 \mathrm{~s}$ from the time of peak enhancement in the ascending aorta [27, 28]. Scan timing is affected by many factors, including cardiac output, injection rate, and the severity of the perfusion abnormality. Therefore, prospective estimation of the optimal scan timing is challenging. In static CTP, stress and rest CTP images are acquired with either prospective ECG triggering or retrospective ECG gating during a single breath-hold. Acquisitions with retrospective ECG gating provide additional information on wall motion during the cardiac cycle. As a result of improved spatial and temporal resolution of CT scanner, there is now potential to simultaneously evaluate both myocardial and coronary artery perfusion by stress static CTP alone using a low effective radiation dose $(2.5 \pm 1.1 \mathrm{mSv})$ [29].

\section{Dynamic CTP imaging}

Dynamic CTP imaging refers to the acquisition of multiple samples during first pass of iodinated contrast in the myocardium (Fig. 3b). Unlike static CTP, the diagnostic performance of this technique is independent of the optimal scan timing and it also allows quantification of myocardial perfusion [30]. The prospective ECG-triggered acquisition in the systolic phase ( $40 \% \mathrm{R}-\mathrm{R}$ interval) is advantageous because this phase is less affected by motion artifact and the hypoenhancement is more visible than in the diastolic phase [31]. Motion in the target phase can be reduced by motion correction algorithms that analyze per-vessel and per-segment motion at the voxel level using information from adjacent cardiac phases within a single cardiac cycle [32]. High temporal resolution and wide detector coverage are desirable to obtain perfusion data for the whole heart. Dual-source CT has high temporal resolution (up to $66 \mathrm{~ms}$ ), which allows acquisition of motion-free images, even at high heart rates. Coronary CTA derived from dynamic CTP imaging by third-generation dual-source $\mathrm{CT}$ is useful for diagnosis of coronary artery stenosis [33]. The ECG-triggered axial shuttle mode available on dual-source $\mathrm{CT}$ allows acquisition of a dynamic CTP dataset for the whole heart en bloc using rapid movement between two table positions. Although the dynamic acquisitions are performed every third or more heartbeat, dynamic CTP with dual-source CT has been reported to have good diagnostic performance for detecting a myocardial perfusion abnormality [34]. Wide-detector CT (e.g., 256 or 320 slice detectors, with $8 \mathrm{~cm} / 16 \mathrm{~cm}$ z-coverage) enables acquisition of data in consecutive heartbeats, providing whole-heart perfusion without temporal gaps [30, 35]. Dynamic CTP imaging by wide-detector CT can accurately quantify myocardial perfusion and is comparable to positron emission tomography (PET) with ${ }^{15} \mathrm{O}$-labelled water, which is the gold standard tracer because it uses a freely diffusible tracer with a $100 \%$ extraction fraction even at high blood flow [36]. The radiation dose required for dynamic CTP is substantially higher than that for static CTP [37]. However, advances in CT scanners, the scan protocol, and reconstruction techniques (e.g., low tube voltage, iterative reconstruction) can reduce radiation exposure $(<4 \mathrm{mSv})$ without impairing image quality [38-40].

\section{CTP scan protocols}

\section{Stress CTP-first, rest CTP-first, or stress CTP-only}

There are two types of CTP protocols, namely stress-first and rest-first (Fig. 5). Rest CTP also serves as CTA. A gap of at least 10-20 min is allowed between stress and rest CTP to reduce the effects of the medication(s) used in the preceding scan [21]. The advantages of stress-first protocol are the higher sensitivity for detecting ischemia than rest-first protocol and optimized CTA at the second acquisition because of the ability to administer medications (e.g., $\beta$-blockers or nitrates) necessary for good image quality without interfering with assessment of stress perfusion. The advantages of rest-first protocol are the higher sensitivity for detecting myocardial infarction than stress-first protocol and the ability to abandon stress CTP if there are no significant lesions in the preceding CTA [25]. However, in the rest-first protocol, contrast contamination from the rest acquisition may hamper the diagnostic performance of the subsequent stress CTP. A rest-first protocol is preferable for patients with a low to intermediate pre-test probability of CAD while a stressfirst protocol is preferable for those with a high pre-test probability of CAD, extensive coronary artery calcifications, or a known history of CAD/PCI or myocardial infarction [23]. Regarding dynamic CTP imaging, a stress-only CTP protocol is often adopted because of the limit of radiation exposure. Although a stress-only CTP protocol cannot yield a quantification of coronary flow reserve (CFR), the diagnostic performance is still high thanks to the routine quantitative technique as mentioned in detail later [34, 35, 41].

\section{Optional scan protocol}

\section{Dual energy CTP}

Dual-energy CT (DECT) refers to acquisition of CT data at two different energy levels. DECT allows for characterization of materials with similar attenuation coefficients but different atomic numbers because the tissues show different attenuation properties at different energy levels [42]. Commonly used DECT techniques include dual-source, rapid $\mathrm{kVp}$ switching, and dual-layer detector technologies [43]. DECT allows generation of multiple additional images, including iodine maps (Fig. 4), and virtual monoenergetic images (VMIs). Iodine maps highlight 


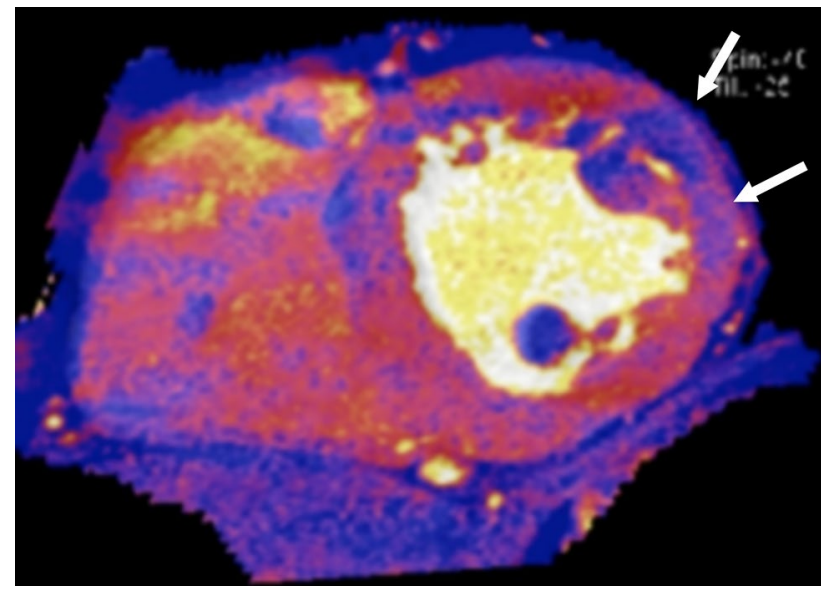

Fig. 4 A dual-energy CT scan overlaid with iodine in the short-axis plane obtained from a static stress myocardial CTP scan showing a perfusion defect in the mid anterolateral septum (arrow). $C T$ computed tomography, CTP computed tomography perfusion

pixels containing iodine and can discriminate normal, ischemic, and infarcted myocardium on stress CTP [44] (Fig. 4). Iodine maps have better diagnostic performance than conventional images [43] and also provide quantitative information [45]. VMIs mimic images obtained at a single energy level and can be generated from 40 to $200 \mathrm{keV}$. Low-energy VMIs (i.e., less than $70 \mathrm{keV}$ ) show higher attenuation of iodine because of approximation with its K-edge. Hence, perfusion defects are more conspicuous due to the enhanced contrast between normal and ischemic myocardium [46]. High-energy VMI is beneficial for reducing beam-hardening artifact [47]. Use of VMI is a balance between image contrast and these artifacts.

\section{Late iodine enhancement CT}

Late iodine enhancement CT (LIE-CT) refers to a delayed phase acquisition following CTP/CTA, which is an optional phase for assessment of myocardial infarction (Fig. 5). As with late gadolinium enhancement MRI (LGE-MRI), iodinated contrast is retained in the extracellular space of scar/ fibrosis [48]. Like LGE-MRI, LIE-CT has the potential for accurate estimation of myocardial viability [48, 49]. LIE-CT is feasible for the assessment of non-ischemic cardiomyopathy such as cardiac sarcoidosis [50]. A large amount of iodine contrast medium is required to clearly visualize LIE. In the protocol including both CTA and CTP, the amount of contrast is enough for LIE-CT [51, 52], while in the protocol including CTA alone, additional injection of contrast medium might be required for LIE-CT [49, 53]. Previously limited by a low contrast-to-noise ratio, LIE-CT has been improved by recent technological advances, such as use of a low tube voltage and iterative reconstruction with a low radiation dose ( $\leq 2 \mathrm{mSv}$ ) [52]. With DECT, low-energy VMI and the iodine map also improve the visibility of myocardial infarction on LIE-CT [53].

\section{Interpretation of CTP images}

Visual assessment is the main evaluation method for static CTP. A perfusion defect is seen as an area with attenuation that is lower than that of remote myocardium in a subendocardial or transmural distribution. The perfusion defect in ischemia could be seen only on the stress CTP images. The perfusion defect in infarction could be seen on both stress and rest images, but the infarction is sometimes missed in the rest images, especially in stress-first protocol. Therefore,
Fig. 5 Comprehensive CTP protocol. In the stress-first protocol, CTP is first acquired with pharmacological stress. The rest CTP is acquired 10-20 min later and also serves as coronary CTA. Nitroglycerin and $\beta$-blockers are administered during this phase to obtain high-quality coronary CTA images. LIE-CT scans can be obtained 5-10 min following the second CTP scan. In the rest-first protocol, rest images are acquired first and stress images are acquired later. $C T A$ computed tomography angiography, CTP computed tomography perfusion, $L I E-C T$ late iodine enhancement computed tomography

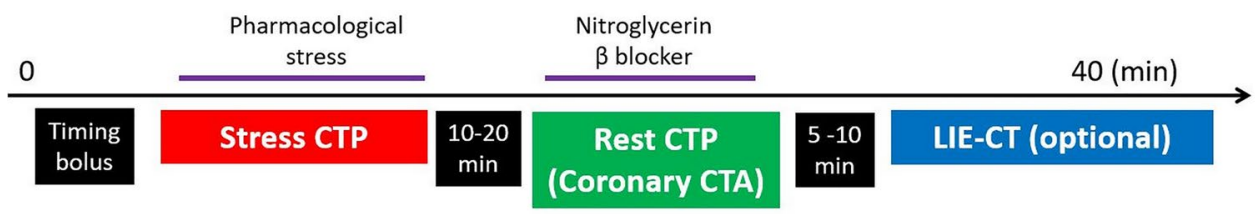

Interchangeable

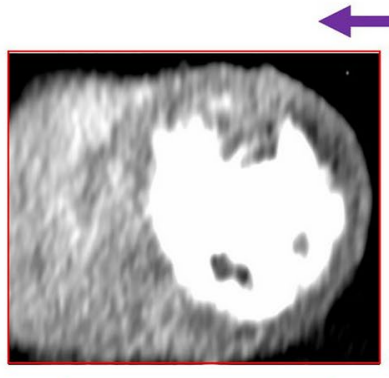

Stress CTP

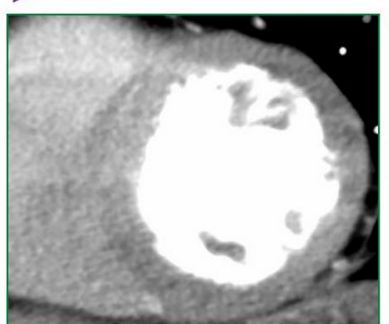

Rest CTP

(Coronary CTA]

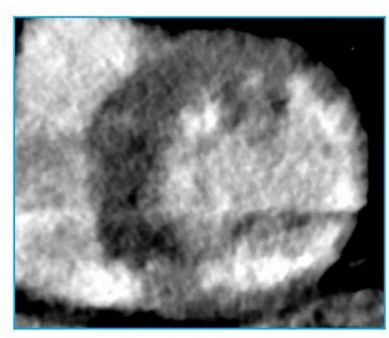

LIE-CT 
LIE-CT is required to identify the infarction [21]. CTP has higher spatial resolution and diagnostic performance for detecting a perfusion abnormality than single-photon emission tomography (SPECT) [54] (Fig. 6, movie in Online Resource 1). The ischemia can be scored semi-quantitatively at a segmental level, similar to SPECT [55]. The American Heart Association recommends that the left ventricle be divided into 17 segments for regional analysis of myocardial perfusion [56]. The scores of the 17 segments can be added to give the summed stress and rest scores, with their difference being the summed difference score [56].

False positives are seen on CTP due to several types of artifact. The most common is beam-hardening artifact, which is caused by preferential attenuation of low-energy photons in a polychromatic X-ray beam. Beam hardening is usually transmural and always occurs in the plane of the $\mathrm{X}$-ray beam adjacent to highly attenuating structures. On
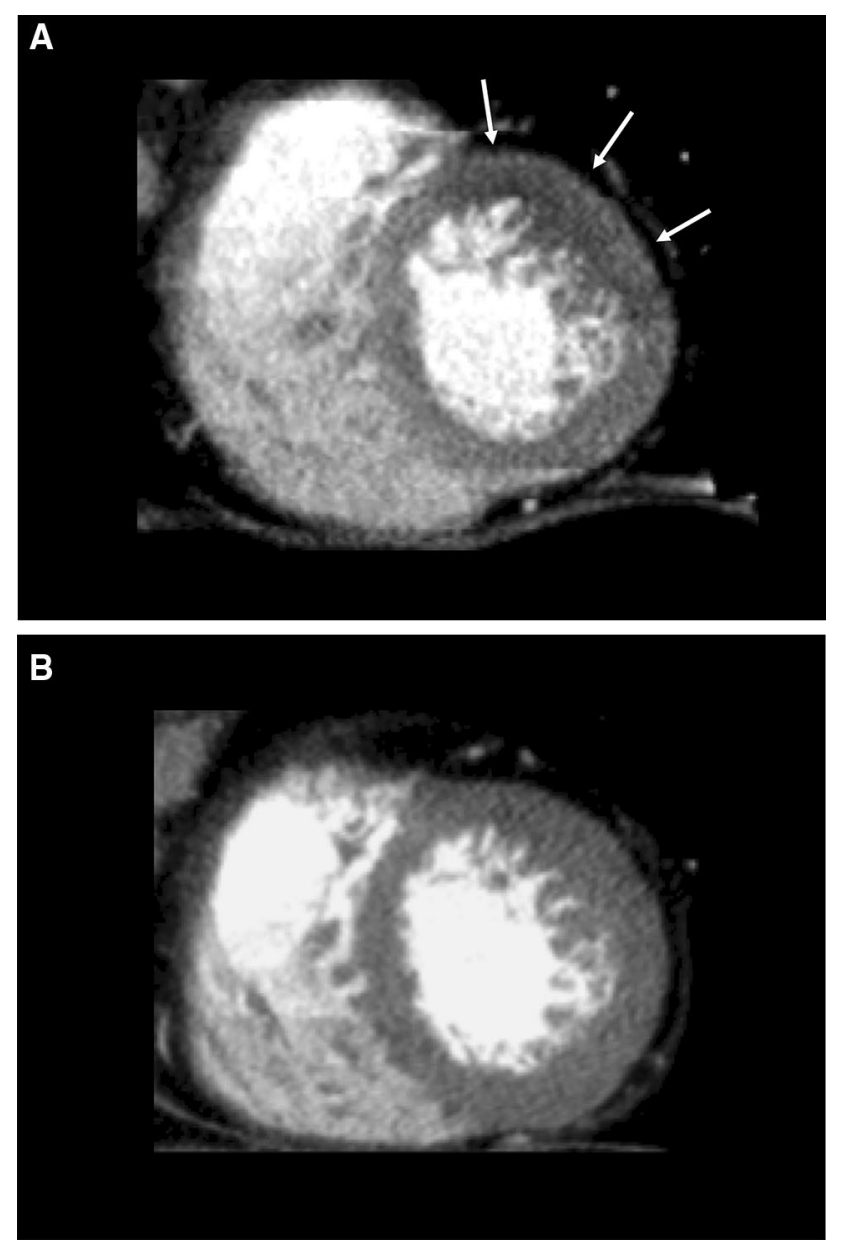

Fig. 6 Static CTP scans for single-vessel disease in a 68-year-old woman with chest pain. She had hypertension and dyslipidemia. a A short-axis CTP image following stress shows a subendocardial perfusion defect in the mid anterolateral wall (arrows). b A rest CTP image in the same position as a shows no perfusion defect, consistent with myocardial ischemia. CTP computed tomography perfusion
CTP, beam-hardening artifact is caused by dense contrast in the left ventricular cavity and descending aorta adjacent to the free wall of the left ventricle and is most commonly seen in the basal inferior and anterior walls of the left ventricle $[21,55]$. These artifacts can be minimized by using dedicated beam-hardening correction algorithms or DECT $[47,57]$. False-positive perfusion defects may also be seen due to cardiac or respiratory motion or reconstruction artifacts, such as cone beam artifact. Misalignment artifacts can produce image stacks acquired during different heartbeats [21]. Semi-quantitative perfusion measurements have been developed for static CTP and include the transmural perfusion ratio (TPR) and myocardial perfusion reserve index (MPRI) [58]. The TPR and MPRI are calculated as follows: $\mathrm{TPR}=$ subendocardial mean attenuation density (AD)/subepicardial mean $\mathrm{AD}$, where $\mathrm{MPRI}=(\mathrm{AD}$ stress $-\mathrm{AD}$ rest $) /$ $\mathrm{AD}$ rest. This semi-quantitative assessment has diagnostic performance comparable to that of visual assessment for detection of obstructive CAD [59].

Dynamic CTP can be assessed visually in the same way as static CTP (movie in Online Resource 2). However, dynamic CTP is mainly assessed by a semi-quantitative or fully quantitative technique, because the visual interpretation of CTP image is more difficult than that of MRI due to the lower image contrast [60]. Semi-quantitative parameters are derived from the myocardial time attenuation curve and include upslope, peak enhancement, time to peak, and area under the curve. Upslope has been shown to have the highest diagnostic performance for detection of a perfusion abnormality [61]. Fully quantitative parameters are derived from both arterial and myocardial time attenuation curves, including myocardial blood flow (MBF), myocardial blood volume, and mean transit time. MBF is the most important quantitative parameter and is frequently used for assessment of perfusion whereas the usefulness of myocardial blood volume and mean transit time is not well studied [62] (Fig. 7). Quantitative parameters are useful for assessment of balanced ischemia in triple-vessel disease, which is difficult to assess on SPECT. There are several mathematical methods for quantifying myocardial perfusion in dynamic CTP, including maximum upslope, a compartment model, an extended Toft model, a Patlak plot, a Fermi parametric model, and model-independent deconvolution [35, 63, 64]. However, the optimal quantification method remains controversial and the cut-off value on dynamic CTP imaging is still not standardized. Recently, relative flow reserve has been shown to be a better alternative to absolute MBF [40, $65,66]$. CFR is calculated as a quantitative ratio of stress MBF to rest MBF in stress and rest dynamic CTP protocol [36]. CFR provides the information of not only epi-coronary artery but also microvascular function, and is significantly impaired due to obstructive epicardial CAD or coronary microvascular dysfunction [67]. Obara et al. reported that 

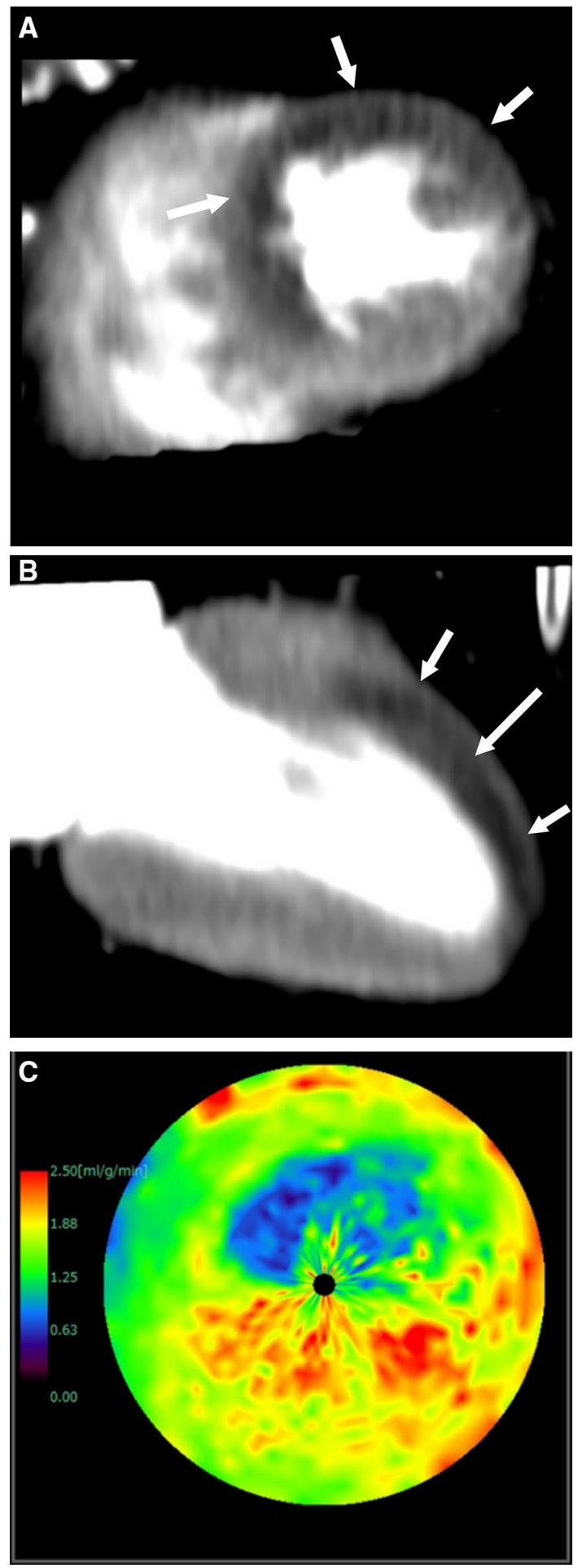

Fig. 7 Dynamic CTP scans for single-vessel disease in an 87-yearold man with chest pain. He had hypertension and a history of smoking. Short-axis (a) and two-chamber (b) grayscale stress dynamic CTP images showing a subendocardial perfusion defect in the anterolateral, anterior, and anteroseptal segments (arrows). c A CT-MBF color-coded image shows that the MBF in the ischemic myocardium is lower than in the remote myocardium. CTP computed tomography perfusion, $C T-M B F$ computed tomography derived-myocardial blood flow
CFR and stress MBF had sufficient sensitivity, PPV, and NPV to detect obstructive CAD assessed by invasive coronary angiography both in per-patient and in per-vessel analyses as with ${ }^{15} \mathrm{O}$-labelled water PET $[68,69]$. Nakamori et al. reported that CFR had the additive value in addition to the stress-rest perfusion MRI for detecting reduced FFR in multivessel disease [70]. van de Hoef et al. reported that discordance between CFR and FFR originated from the involvement of the microvascular function, and the risk for major adverse cardiac events associated with FFR/CFR discordance was mainly attributable to stenoses with abnormal CFR [71]. Although CFR is very useful as diagnostic and prognostic predictor, stress and rest dynamic CTP protocol has restriction of use due to the radiation exposure. Therefore, further radiation reduction of dynamic CTP is required to use CFR in clinical routine.

\section{Iterative reconstruction and other algorithms for CTP}

Radiation exposure and suboptimal image contrast are important concerns in CTP imaging. Iterative reconstruction algorithms decrease image noise, allowing use of low-dose CT techniques (e.g., low tube voltage) with maintenance of image quality [72, 73]. There are several iterative reconstruction (IR) algorithms; some are hybrid IR" techniques that are combined with filtered back projection (FBP) to reduce reconstruction time and some are "fully IR" with higher performance [74]. Use of a fully IR technique improves image quality without altering hemodynamic parameters on low-dose dynamic CTP when compared with FBP or hybrid IR [39]. Recently, artificial intelligence was used to improve CT image reconstruction [75]. These techniques have the potential to optimize the quality of low-dose myocardial CTP images with shortening of reconstruction times [76, 77].

\section{Advantages of CTP}

A major advantage of CTP is that it allows a perfusion defect to be visualized directly with high temporal and spatial resolution, as would MRI [78]. CTP is useful for assessment of the hemodynamic significance and effective classification of coronary lesions, allowing integrated evaluation of CAD when combined with CTA (Fig. 8) [30, 79]. Moreover, myocardial perfusion can be quantified and the effect of treatment after revascularization can be evaluated [35, 80]. CTP can provide functional information even in patients with heavy calcifications or stenting, which are limitations of CT-FFR [41, 81]. CTP is preferred by patients [82] and is more cost-effective than SPECT [83]. 
Fig. 8 Dynamic CTP scans for triple-vessel disease in an 84-year-old woman with chest pain. a Short-axis view of a dynamic CTP (grayscale) shows a subendocardial perfusion defect in the entire circumference of the heart (white arrows). b A CT-MBF color-coded image shows low CT-MBF throughout the heart. SPECT during $\mathbf{c}$ stress and $\mathbf{d}$ at rest shows a reversible perfusion defect in the lateral wall but no marked perfusion defect in the other regions. Invasive coronary angiography of the e right and f left coronary arteries shows severe triple-vessel disease. In this case, single-photon emission tomography did not accurately detect the presence of triple-vessel disease, known as balanced ischemia. CTP computed tomography perfusion, $C T-M B F$ computed tomography derived-myocardial blood flow, SPECT single-photon emission computed tomography
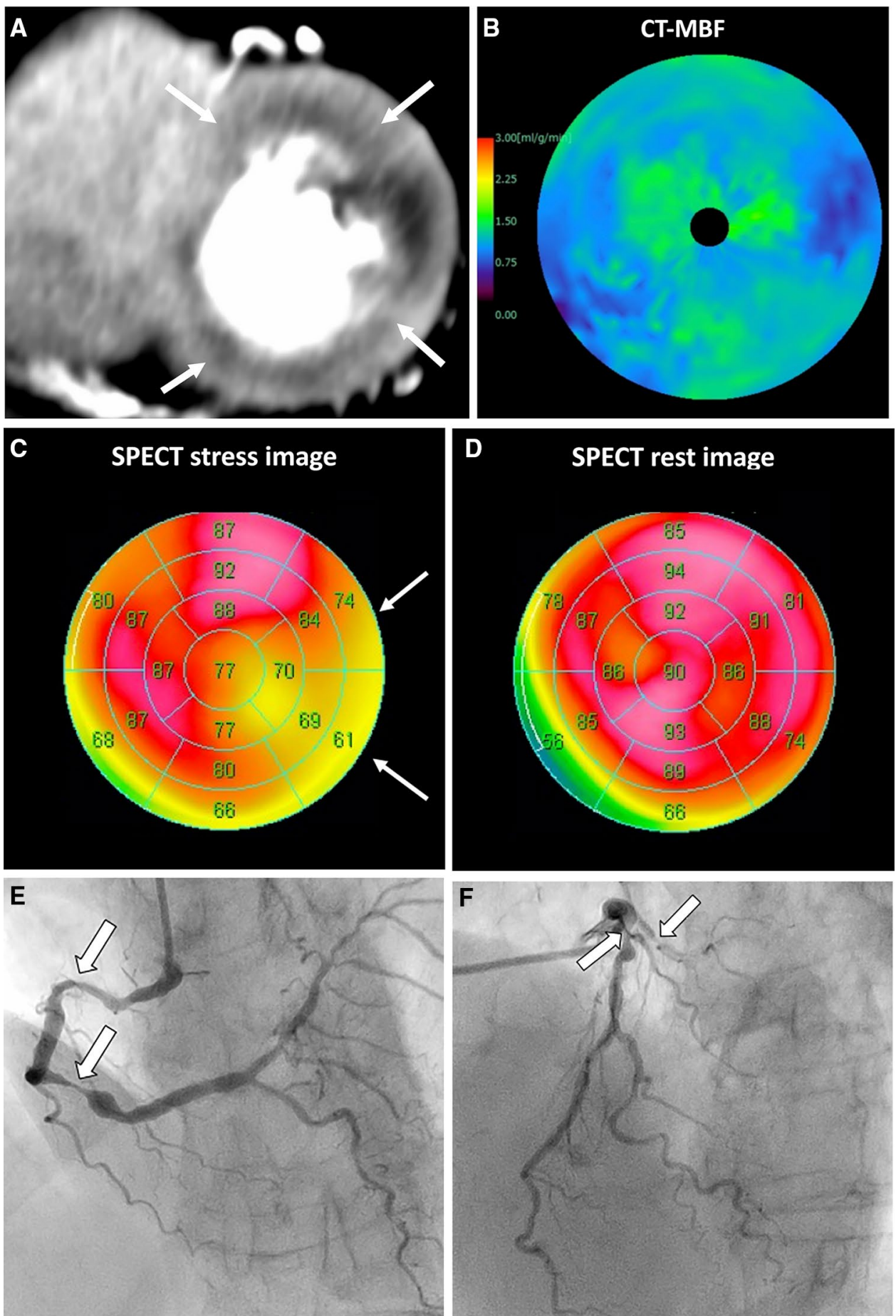

\section{Limitations of CTP}

CTP is not widely available because it requires a high level of expertise and multiple resources, including advanced scanners and reconstruction algorithms. Greater radiation exposure, use of a higher contrast dose compared with CTA alone (static CTP alone 1.9-15.7 mSv, dynamic CTP alone 3.8-12.8 $\mathrm{mSv}$, combined CTA and stress CTP protocol 3-16 mSv) [37, 84], and side effects of medications are also issues. Static CTP is limited in evaluation of patients after CABG because of the complexity of myocardial perfusion via the native coronary arteries and bypass grafts. 


\section{Current evidence of CTP}

Multiple studies have established the high diagnostic performance of both static and dynamic CTP for detecting hemodynamically significant stenosis $[34,36,41,61,62$, 78, 81, 84-90] (Table 2). Recent meta-analyses indicated that dynamic CTP has higher sensitivity but lower specificity than static CTP (sensitivity, $85 \%$ vs $72-80 \%$; specificity, $90-93 \%$ vs $81-83 \%$ ) [84, 90] (Table 2). Moreover, the CORE320 study $(n=381)$ demonstrated that static CTP has incremental diagnostic value over CTA for detection of hemodynamically significant coronary lesions, with a specificity of $74 \%$ vs $51 \%$ for CTA, a PPV of $65 \%$ vs $53 \%$, and an area under the receiver-operating characteristic curve (AUC) of 0.87 vs 0.84 [91]. Pontone et al. $(n=147)$ reported similar results in static CTP (a specificity of $95 \%$ vs $76 \%$ for CTA and a PPV of $87 \%$ vs $61 \%$ ) [92]. The CRESCENT-II trial indicated that incorporation of dynamic CTP imaging as part of a tiered diagnostic approach could improve the clinical value and efficiency of cardiac CT in the diagnostic work-up of patients with stable CAD and was an effective alternative to standard guideline-directed functional testing [93]. Both static and dynamic CTP has also been reported to have incremental predictive value over clinical risk factors and CTA in assessment for future major adverse cardiac events (MACE) [94, 95]. Dynamic CTP was shown to have higher prognostic value for MACE than CTA and CT-FFR, independent of clinical risk factors [96].

\section{CT-fractional flow reserve}

\section{Principle of CT-FFR}

CT-FFR calculates the FFR from coronary CTA data at rest using computational fluid dynamics to generate a mathematical model of coronary flow, pressure, and resistance [97]. This modeling relies on the following four important principles and assumptions: (1) under resting conditions, the total coronary flow is proportional to myocardial mass; (2) resting coronary microvascular resistance is inversely proportional to the size of the epicardial coronary arteries; (3) the dilatory response of the coronary arteries to adenosine during ICA is predictable and can be used to create a computational model of the maximal hyperemic state, which is generally simulated by reducing the microvascular resistance by a factor of 0.21 , and although adenosine is not required, administration of nitroglycerine is a pre-requisite for measurement of CT-FFR; and (4) solving the complex three-dimensional Navier-Stokes equation that governs fluid dynamics can compute the flow and pressure across the coronary vascular bed [98].

Table 2 Diagnostic performance of computed tomography perfusion with invasive fractional flow reserve as the gold standard

\begin{tabular}{|c|c|c|c|c|c|c|c|c|}
\hline Study & Patients, $n$ & Technique & $\begin{array}{l}\text { Sensitivity } \\
(\%)\end{array}$ & Specificity (\%) & PPV (\%) & NPV (\%) & AUC & MBF cut-off \\
\hline Bettencourt et al. [78] & 101 & Static & 55 & 95 & 78 & 87 & 0.75 & \\
\hline Yang et al. [86] & 75 & Static & 80 & 95 & 92 & 87 & 0.87 & \\
\hline Yang et al. [87] & 72 & Static & 79 & 91 & 86 & 87 & 0.88 & \\
\hline Ihdayhid et al. [41] & 46 & Static & 54 & 92 & 79 & 77 & 0.72 & \\
\hline Greif et al. [62] & 65 & Dynamic & 95 & 74 & 49 & 98 & 0.71 & $0.75 \mathrm{ml} / \mathrm{g} / \mathrm{min}$ \\
\hline Huber et al. [61] & 32 & Dynamic & 76 & 100 & 100 & 91 & 0.86 & $1.64 \mathrm{ml} / \mathrm{g} / \mathrm{min}$ \\
\hline Rossi et al. [88] & 80 & Dynamic & 88 & 90 & 77 & 95 & 0.95 & $0.78 \mathrm{ml} / \mathrm{g} / \mathrm{min}$ \\
\hline Coenen et al. [34] & 43 & Dynamic & 75 & 78 & 78 & 75 & 0.78 & $0.76 \mathrm{ml} / \mathrm{g} / \mathrm{min}$ \\
\hline Coenen et al. [81] & 74 & Dynamic & 75 & 61 & 63 & 73 & 0.78 & $0.91 \mathrm{ml} / \mathrm{g} / \mathrm{min}$ \\
\hline Meta-analysis & Vessels, $n$ & \multicolumn{2}{|c|}{ Protocol } & Sensitivity (\%) & Specificity (\%) & PLR & NLR & AUC \\
\hline Takx et al. [85] & 1074 & \multicolumn{2}{|c|}{ Static } & 78 & 86 & 5.74 & 0.22 & 0.91 \\
\hline Lu et al. [89] & 697 & \multicolumn{2}{|c|}{ Dynamic } & 85 & 81 & 4.46 & 0.21 & 0.91 \\
\hline \multirow[t]{3}{*}{ Celeng et al. [90] } & 2118 & \multicolumn{2}{|c|}{ Overall } & 81 & 86 & 6.28 & 0.23 & \\
\hline & & \multicolumn{2}{|c|}{ Static } & 72 & 90 & & & \\
\hline & & \multicolumn{2}{|c|}{ Dynamic } & 85 & 81 & & & \\
\hline \multirow[t]{3}{*}{ Hamon et al. [84] } & 2336 & \multicolumn{2}{|c|}{ Overall } & 82 & 89 & 7.72 & 0.21 & 0.94 \\
\hline & & \multicolumn{2}{|c|}{ Static } & 80 & 93 & 10.77 & 0.23 & 0.96 \\
\hline & & \multicolumn{2}{|c|}{ Dynamic } & 85 & 83 & 4.89 & 0.17 & 0.94 \\
\hline
\end{tabular}

$A U C$ area under the curve, $M B F$ myocardial blood flow, $N L R$ negative likelihood ratio, $N P V$ negative predictive value, $P L R$ positive likelihood ratio, $P P V$ positive predictive value 


\section{Technology of CT-FFR}

Several large-scale multicenter trials have shown the high diagnostic performance of CT-FFR by a remote analysis service in selected patients with CAD [98-100]. In this technology, the CTA data are transferred through a secure network to an off-site location. A patient-specific three-dimensional model of the coronary arteries is created after segmentation of the CTA data. Using the above-mentioned mathematical assumptions, a supercomputer performs complex post-processing to solve the equations governing fluid dynamics and blood flow [97, 101]. Simulated hyperemic blood flow and pressure data are then generated, and the results are sent back to the referring institution within a few hours (Fig. 9). On-site vendor-based platforms are also available in some institutions, including a machine learning-based algorithm [102, 103], four-dimensional CT image tracking (registration) and structural and fluid analysis $[104,105]$, and patientspecific lumped parameter models [106, 107].

However, the off-site CT-FFR using a remote analysis service is recently received with national reimbursement approval in Japan, but the available facilities are strictly limited by requirements. Meanwhile, the on-site CT-FFR is also available only for clinical research.

\section{Interpretation of CT-FFR}

CT-FFR is presented as a color-coded map of continuous CT-FFR values computed along each coronary vessel (Fig. 10). These values are both specific for a lesion and for the entire coronary tree. CT-FFR results are interpreted in conjunction with anatomic CTA findings, including vessel size, presence and location of stenosis, suitability for revascularization, and other CT-FFR values. Coronary stenosis with a precipitous drop in CT-FFR across the lesion, particularly if $<0.75$, is associated with lesion-specific ischemia. A CT-FFR value $>0.8$ distal to a stenosis is rarely associated with ischemia. A CT-FFR value between 0.75 and 0.80 is "gray zone" or borderline [101]. It is important to measure the CT-FFR immediately distal to a stenotic vessel because the CT-FFR value in the most distal vessel segment may not necessarily correlate with the functional significance of the stenosis. A gradual drop in pressure along the length of the vessel without focal stenosis, particularly for borderline values, can be a normal phenomenon or due to a small vessel size, inadequate response to a nitrate, diffuse disease, or a serial lesion $[97,101]$. There are ICA data showing that diffuse disease can cause a hemodynamically significant drop in the pressure gradient [108] but the CT data are inadequate. The referring clinician should interpret the findings in view of the anatomy, entire physiological model, and symptoms.

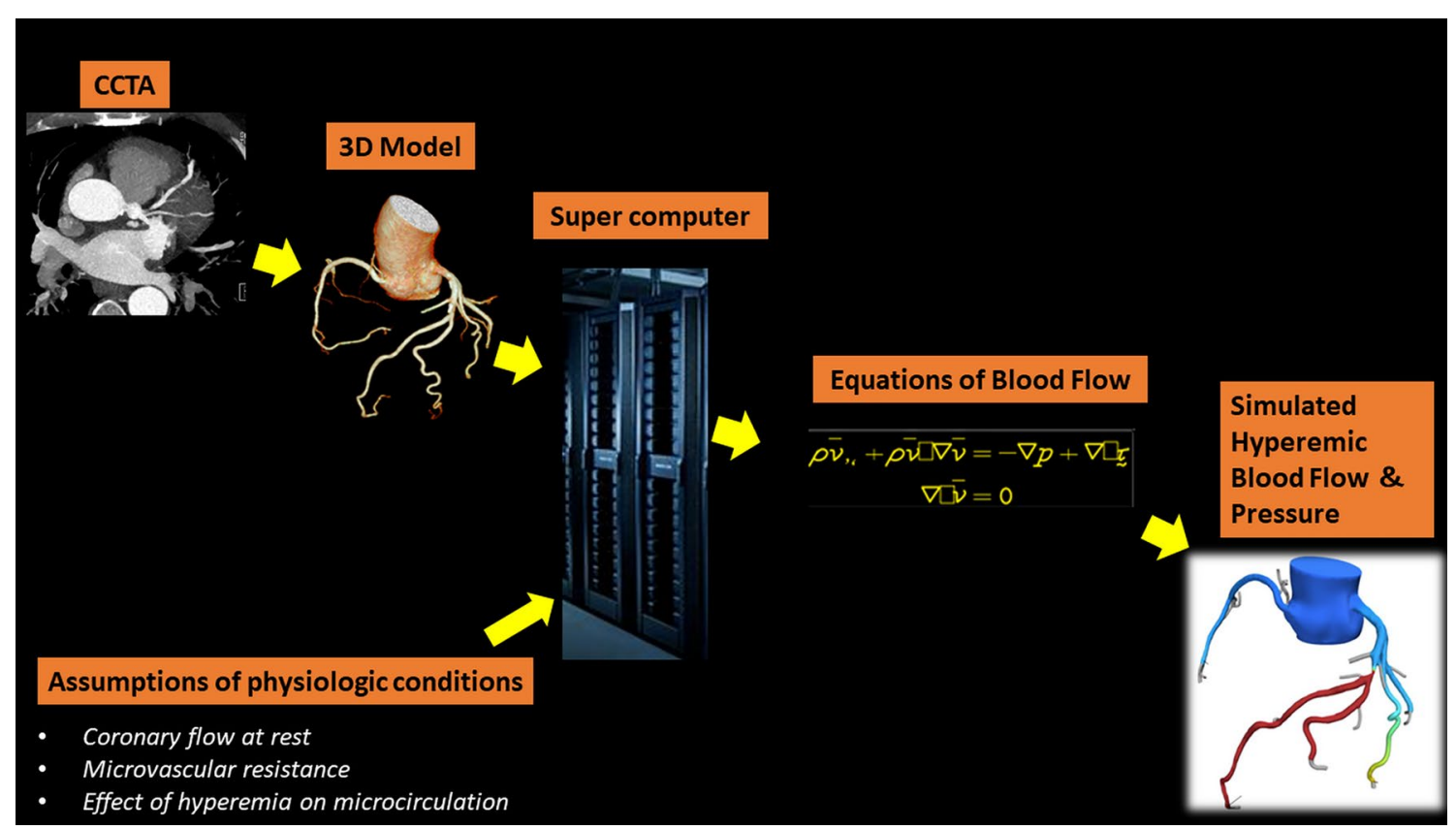

Fig. 9 Illustration showing the CT-FFR technique. The CCTA data are segmented and a three-dimensional model is generated. This model is then processed by a supercomputer using assumptions of physiological conditions to solve the Navier-Stokes equation and generate a hyperemic model of coronary flow and pressure. CCTA coronary computed tomography angiography, $C T$ computed tomography, FFR fractional flow reserve 
Summary: $F F R_{C T}$ is $>0.80$ in all coronary vessels.
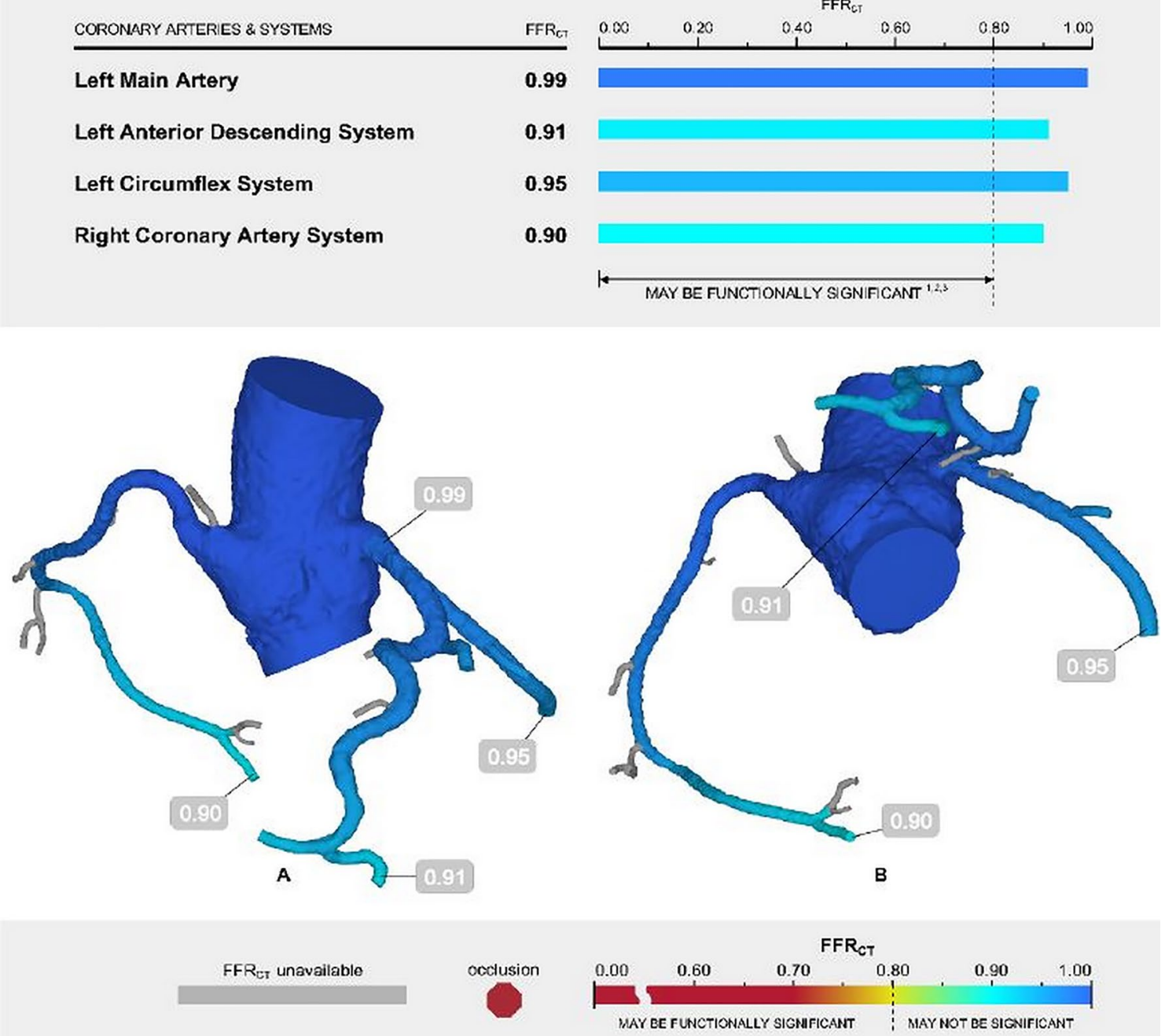

Fig. 10 Normal CT-FFR report for a 59-year-old man with atypical chest pain. The CT-FFR report shows the coronary arteries colorcoded according to their CT-FFR values. In this patient, all the major

\section{Clinical utility: when to use CT-FFR?}

CT-FFR is most valuable in patients with moderate $(50-70 \%)$ stenosis on CTA. CT-FFR clarifies the hemodynamic significance of stenosis in these patients and aids decision-making [109]. If a patient has moderate coronary artery stenosis on CTA and the CT-FFR value is $>0.8$, ICA can be avoided (Fig. 11). However, if the value is $<0.8$, ICA can be performed with the intention to treat the lesion (Fig. 12). If CTA shows a non-obstructive lesion $(<50 \%$ stenosis), the patient is referred for medical treatment and there is no need to perform CT-FFR or ICA. If CTA shows severe stenosis, the patient is referred for ICA without the need for CT-FFR. In patients with multivessel disease or coronary arteries show normal values $(>0.8) . C T$ computed tomography, FFR fractional flow reserve

tandem lesions, CT-FFR helps to identify the lesions needing revascularization [110]. However, CT-FFR may underestimate the contribution of true stenosis in serial stenoses [111]. A noninvasive PCI planning tool has been devised to help determine the contribution of true stenosis in serial and diffuse CAD [111]. A virtual stent can also be placed and the response to revascularization estimated [112].

\section{Current evidence of CT-FFR}

The accuracy of CT-FFR has been validated in several studies (Table 3) [81, 98-100, 103, 105-107, 113-122], predominantly with off-site technology, which uses invasive FFR as the gold standard. The specificity of CT when 


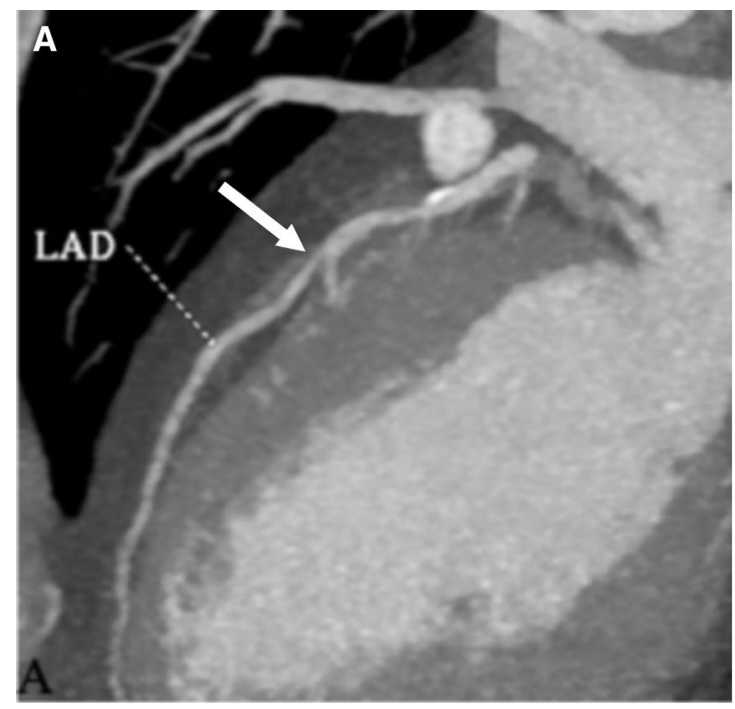

Fig. 11 a A curved multiplanar reconstruction image of the LAD in a 52-year-old man with chest pain showing moderate stenosis of the mid LAD (arrow). b CT-FFR in the same patient shows a value of 0.89 in the mid LAD, which is within normal limits, indicating that
B

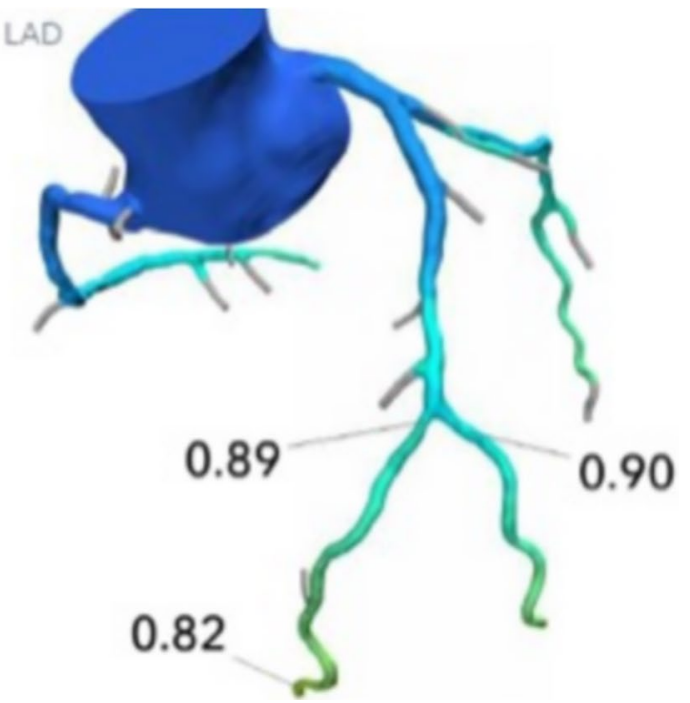

there is no lesion-specific ischemia. The patient was referred for medical management. Therefore, CT-FFR helped to avoid ICA. $C T$ computed tomography, FFR fractional flow reserve, ICA invasive coronary angiography, $L A D$ left anterior descending artery
Fig. 12 a A curved multiplanar reconstruction image of the LAD in a 66-year-old woman showing a non-calcified plaque in the proximal LAD causing moderate luminal stenosis (arrow). b CT-FFR shows a value of 0.76 in the proximal LAD, which is indicative of hemodynamically significant stenosis. c The patient was referred for ICA, which confirmed a significant stenosis with a decreased FFR of 0.65 . $C T$ computed tomography, FFR fractional flow reserve, $I C A$ invasive coronary angiography, $L A D$ left anterior descending artery
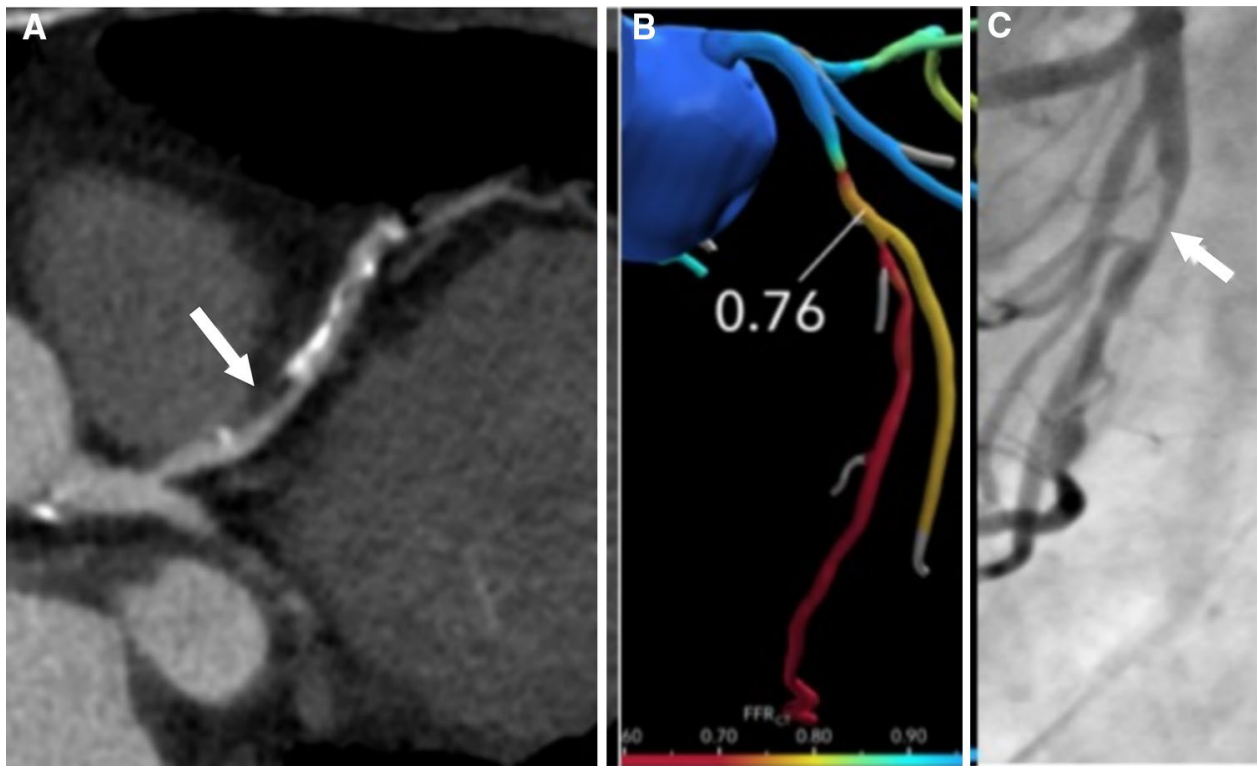

CT-FFR is used is better than when CTA alone is used [82\% vs $40 \%$ in DISCOVER-FLOW (per-vessel), 54\% vs $42 \%$ in DEFACTO (per-patient), and $79 \%$ vs $34 \%$ in NXT (per-patient)] [98-100]. Similar results were seen with onsite vendor-based and machine learning algorithms [81, 103, 105-107, 113, 116-119]. One meta-analysis showed a pooled specificity of $76 \%$ and an odds ratio of 26.2 for detecting ischemic lesions on the per-patient basis [121], whereas another study showed that CT-FFR had higher specificity than CTA (71\% vs 39\%) but similar sensitivity $(91 \%)$ on the per-patient basis [122]. CT-FFR had diagnostic accuracy similar to that of SPECT but had higher sensitivity for predicting FFR-guided revascularization [114]. With good-quality CTA images, CT-FFR was found to have higher diagnostic performance than CTA, SPECT, or PET on a pervessel basis whereas PET had favorable performance on perpatient and intention-to-diagnose analysis [115].

The studies for CT-FFR are summarized in Table 4. The ability of CT-FFR to evaluate lesion-specific ischemia with high specificity makes it an effective gatekeeper for ICA. In NXT, $68 \%$ of false positives were reclassified as true negatives [100]. PLATFORM, which was a clinical utility trial, 
Table 3 Diagnostic performance of computed tomography-fractional flow reserve with invasive fractional flow reserve as the gold standard

\begin{tabular}{|c|c|c|c|c|c|c|c|c|c|c|}
\hline Study & System & \multicolumn{2}{|c|}{ Study type } & Basis & Accuracy $(\%)$ & $\begin{array}{l}\text { Sensi- } \\
\text { tivity } \\
(\%)\end{array}$ & $\begin{array}{l}\text { Speci- } \\
\text { ficity } \\
(\%)\end{array}$ & PPV $(\%)$ & NPV (\%) & AUC \\
\hline $\begin{array}{l}\text { Koo et al. (DISCOVER- } \\
\text { FLOW) [98] }\end{array}$ & Off-site & \multicolumn{2}{|c|}{ Prospective multicenter } & 159 vessels & 84 & 88 & 82 & 74 & 92 & 0.90 \\
\hline Min et al. [DEFACTO) [99] & Off-site & \multicolumn{2}{|c|}{ Prospective multicenter } & 407 vessels & 69 & 80 & 63 & 56 & 84 & $\mathrm{NA}$ \\
\hline Nørgaard et al. (NXT) [100] & Off-site & \multicolumn{2}{|c|}{ Prospective multicenter } & 484 vessels & 86 & 84 & 86 & 61 & 95 & 0.93 \\
\hline $\begin{array}{l}\text { Sand et al. (ReASSESS) } \\
\text { [114] }\end{array}$ & Off-site & \multicolumn{2}{|c|}{ Prospective single-center } & 143 patients & 70 & 91 & 55 & 58 & 90 & NA \\
\hline Driessen et al. [115] & Off-site & \multicolumn{2}{|c|}{ Prospective single-center } & 505 vessels & 87 & 90 & 86 & 65 & 96 & 0.94 \\
\hline Coenen et al. [81] & On-site & \multicolumn{2}{|c|}{ Retrospective two-center } & 142 vessels & 70 & 82 & 60 & 65 & 79 & 0.78 \\
\hline De Geer et al. [116] & On-site & \multicolumn{2}{|c|}{ Retrospective single-center } & 23 vessels & 78 & 83 & 76 & 56 & 93 & NA \\
\hline Fujimoto et al. [105] & On-site & \multicolumn{2}{|c|}{ Retrospective two-center } & 104 vessels & 84 & 91 & 78 & 76 & 92 & 0.85 \\
\hline Donnelly et al. [106] & On-site & \multicolumn{2}{|c|}{ Prospective two-center } & 60 vessels & 78 & 91 & 72 & 63 & 93 & 0.89 \\
\hline Kim et al. [117] & Off-site & \multicolumn{2}{|c|}{ Prospective multicenter } & 48 vessels & 77 & 85 & 57 & 83 & 62 & NA \\
\hline Renker et al. [113] & On-site & \multicolumn{2}{|c|}{ Retrospective single center } & 67 vessels & 85 & 85 & 85 & 71 & 93 & 0.92 \\
\hline Wardziak et al. [118] & On-site & \multicolumn{2}{|c|}{ Retrospective single center } & 96 vessels & 74 & 76 & 72 & 67 & 80 & 0.84 \\
\hline van Hamersvelt et al. [107] & On-site & \multicolumn{2}{|c|}{ Retrospective single-center } & 77 vessels & 83 & 89 & 78 & 79 & 89 & 0.87 \\
\hline Coenen et al. [103] & On-site & \multicolumn{2}{|c|}{ Retrospective multicenter } & 525 vessels & 78 & 81 & 76 & 70 & 85 & 0.84 \\
\hline Kurata A et al. [119] & On-site & \multicolumn{2}{|c|}{ Retrospective multicenter } & 91 vessels & 82 & 89 & 75 & 79 & 87 & 0.91 \\
\hline Meta-analysis & \multicolumn{2}{|c|}{$\begin{array}{l}\text { Total number of } \\
\text { studies }\end{array}$} & Vessels & OR & e) & $\begin{array}{l}\text { pecificity } \\
\%)\end{array}$ & PLR & & NLR & AUC \\
\hline Baumann et al. [120] & 5 & & 1306 & 84 & 7 & 5 & NA & & NA & 0.90 \\
\hline Wu et al. [121] & 7 & & 1377 & 6.87 & 7 & 6 & 3.51 & & 0.21 & 0.86 \\
\hline Danad et al. [122] & 3 & & 1050 & .15 & 7 & 8 & 4.02 & & 0.22 & 0.92 \\
\hline
\end{tabular}

$A U C$ area under the curve, $D O R$ diagnostic odds ratio, $F F R$ fractional flow reserve, $N A$ not available, $N L R$ negative likelihood ratio, $N P V$ negative predictive value, $P L R$ positive likelihood ratio, $P P V$ positive predictive value

showed that use of CT-FFR resulted in cancellation of ICA in $61 \%$ of patients in whom it had been planned based only on CTA findings without any adverse events at the 90-day follow-up [123]. By using CT-FFR, the overall incidence of non-obstructive disease on ICA decreased to $12 \%$ from $73 \%$ (a decrease of $61 \%$ ) [123]. At the 12-month followup, adverse events were infrequent (only 5 of 581 followed cases). In the planned invasive stratum, mean costs were 33\% lower with CTA and CT-FFR [124]. Data are also emerging on the use of CT-FFR in decision-making and outcomes. The FFR ${ }_{\text {CT }}$ RIPCORD study showed that use of CT-FFR resulted in a change in the management plan (medical vs PCI vs $\mathrm{CABG}$ ) in $36 \%$ of patients. There was a $30 \%$ reduction in PCI, an $18 \%$ change in the target vessel, and reassignment from optimal medical therapy to PCI in $12 \%$ of cases [125]. The SYNTAX III Revolution trial showed that CTFFR aids decision-making without need for ICA in patients with left main or 3-vessel CAD. A decision-making based on CTA and CT-FFR showed high agreement with the decision derived from ICA [126]. This study also showed that the addition of CT-FFR to CTA alone changed the treatment decision (between PCI and CABG) in 7\% of the patients and modified selection of vessels for revascularization in
$12 \%$ [127]. Using CT-FFR resulted in 12\% fewer MACE at 1 year and 30\% lower costs with improved quality of life in comparison with ICA and visual guidance [128]. There was no MACE at 1 year in patients in whom ICA was deferred as a consequence of a negative CT-FFR, indicating that this is a safe and feasible test $[113,125]$. The 1-year outcomes in the ADVANCE $\mathrm{FFR}_{\mathrm{CT}}$ registry study also indicated a low event rate, fewer MACE, and less revascularization in those with negative CT-FFR [129].

CT-FFR shows promise in the evaluation of biomechanical forces on atherosclerotic plaques, which play a role in their development and progression [130,131]. CT-FFR can also aid in the prediction of acute coronary syndrome with potentially superior accuracy (area under the curve, 0.725) on CTA [132].

\section{Limitations of CT-FFR}

CT-FFR relies on high-quality CTA images. Therefore, it is critical to adhere to a rigorous guideline-driven protocol [133] with maximal coronary vasodilation and without image noise, motion, or misalignment artifacts [101, 134]. Inadequate contrast opacification and calcium blooming can 


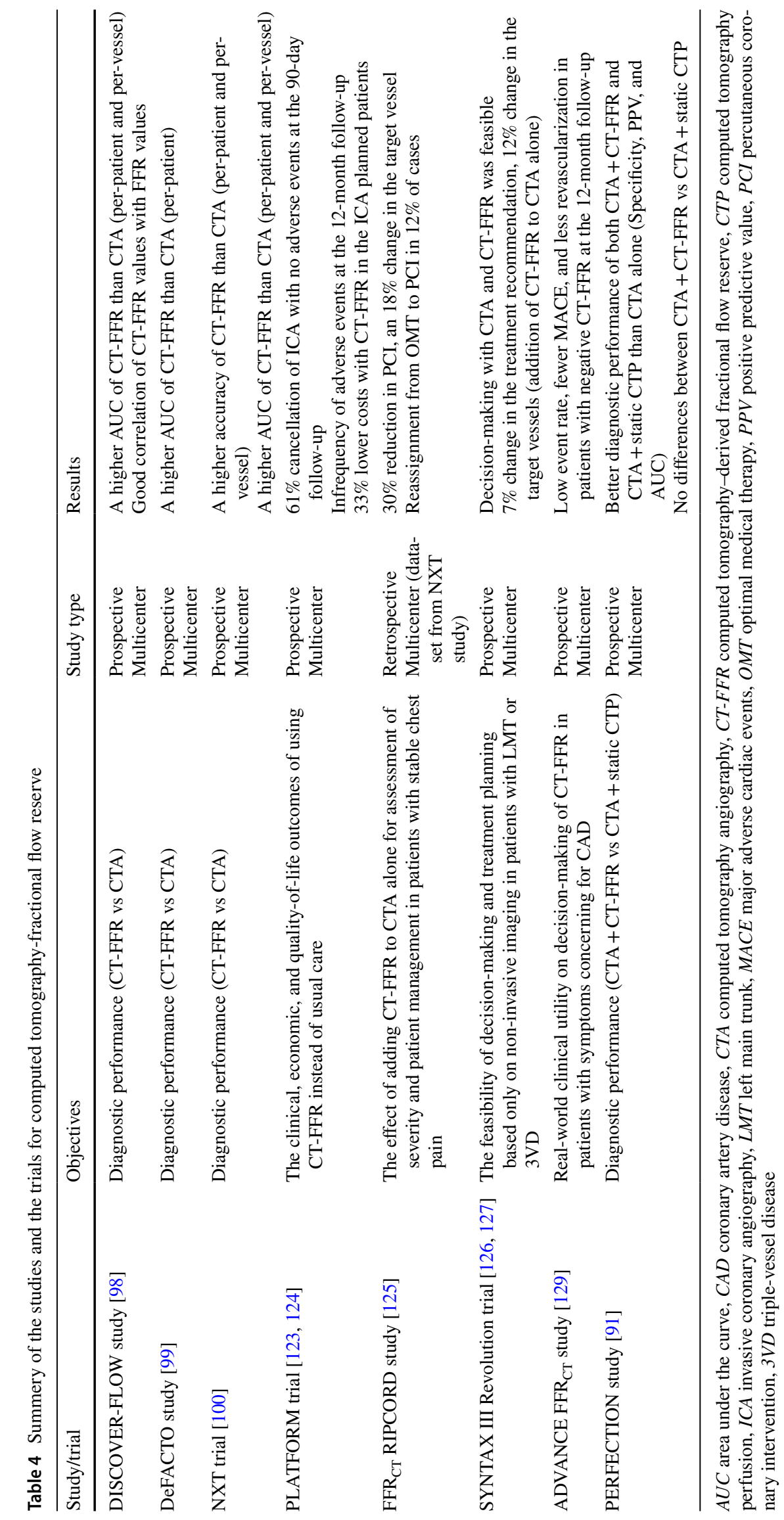


also negatively affect CT-FFR evaluation [135]. However, recent studies have shown that the performance of CT-FFR in patients with high coronary calcium score is superior to that of CTA alone $[130,136]$. The rates of rejection on CTFFR analysis in the ADVANCE registry and in a large clinical cohort were $2.9 \%$ and $8.4 \%$, respectively [137]. CT-FFR is currently not suitable for patients with stenting or CABG. CT-FFR is also not effective in patients with unstable angina and performs modestly in detecting ischemia in non-culprit lesions in patients with STEMI, probably because of the vessel volume is smaller in these patients than in those with stable angina, which confounds the assumption that size is related to resistance [138]. As of now, CT-FFR is not ideal for use in the emergency setting because of the abovementioned inadequate assumptions. A recent study showed that although CT-FFR has good accuracy overall (81.0\%), it has poor accuracy $(46.1 \%)$ in the borderline CT-FFR range (0.7-0.8) [139]. Therefore, the results of CT-FFR should be interpreted cautiously in the context of patient-specific risk factors. Furthermore, there are data that show CT-FFR to be abnormal in up to $16.6 \%$ of patients with insignificant stenosis $(<50 \%)$ and conversely that it can be normal in 50\% of patients with moderate stenosis [140, 141]. Recent studies have cast doubt on the reproducibility of CT-FFR [138]. For example, there is not a perfect match between the CT-FFR and invasive FFR values [139]. This could be because the pressure sensor on invasive FFR is not at the exact location where CT-FFR was measured [101] or the dose of nitroglycerin before CTA was inadequate or nitroglycerin was not administered at all [101].

\section{Which $\mathrm{CT}$ imaging technique is best for ischemia?}

The choice between CTP and CT-FFR depends on the availability of the technology and expertise (Table 5). CTP requires a high-end scanner, ideally with single heartbeat coverage and dedicated personnel, protocols, and postprocessing. CT-FFR can be obtained from CTA data from any scanner, but the images must be of high quality, the results are not available immediately, and there is an additional cost for using off-site CT-FFR technology [142]. Onsite systems are not commercially available as yet. CT-FFR is better than CTP for identifying patients with balanced ischemia, multi-vessel disease, or serial lesions who will benefit from revascularization [98] but is of limited value in patients with unstable angina, especially for non-culprit lesions in those with a recent STEMI. CTP is better than CT-FFR in patients with heavy calcifications or stenting $[143,144]$. Coenen et al. showed that the diagnostic performance of CTP and CT-FFR was comparable for identifying functionally significant CAD assessed by invasive FFR [81] (Fig. 13). A similar study showed that CT-FFR and CTP had comparable performance, with either test improving the performance of CTA [87]. A meta-analysis also showed that the pooled specificity values for CTP $(0.77)$ and CT-FFR (0.72) were higher than that of CTA (0.43) [145]. Otherwise, Li et al. showed that CTP outperformed CT-FFR for identifying lesions causing ischemia assessed by invasive coronary angiography and FFR [146]. The PERFECTION study showed that both CTA + CT-FFR and CTA + CTP have

Table 5 Comparison of the various computed tomography techniques used to evaluate myocardial ischemia

\begin{tabular}{lll}
\hline & Advantages & Disadvantages \\
\hline Coronary CTA & $\begin{array}{l}\text { Visualization of coronary artery stenosis and plaque morphol- } \\
\text { ogy }\end{array}$ & Unassessable segments (artifact, calcification) \\
& Widely available in clinical practice & Low PPV for detecting myocardial ischemia \\
CT-FFR & CTA anatomy- and CFD-based functional assessment & Depends on image quality of coronary CTA \\
& No scan additional to coronary CTA & Appropriate patient selection (image-related, patient-related \\
& High diagnostic performance & factors influencing CT-FFR calculation) \\
& Effective modification to coronary CTA based decision-making & On-site analysis (requiring a learning period, objectivity) \\
& & Less information on the stenosis-related territory \\
CTP & High spatial resolution & Radiation exposure and contrast dose additional to coronary \\
& Real-time stress myocardial perfusion imaging & CTA \\
Visualization of myocardial ischemia (area and transmural & Risk of side effects from the vasodilator agent \\
& extent) & Long examination time (30-60 min) \\
& Quantification (CT-MBF using dynamic CTP) & \\
Incremental value to coronary CTA & \\
\hline
\end{tabular}

$C F D$ computational flow dynamics, $C T A$ computed tomography angiography, $C T$ - $F F R$ computed tomography-derived fractional flow reserve, $C T-M B F$ computed tomography derived myocardial blood flow, $C T P$ computed tomography perfusion, $P C I$ percutaneous coronary intervention 
A

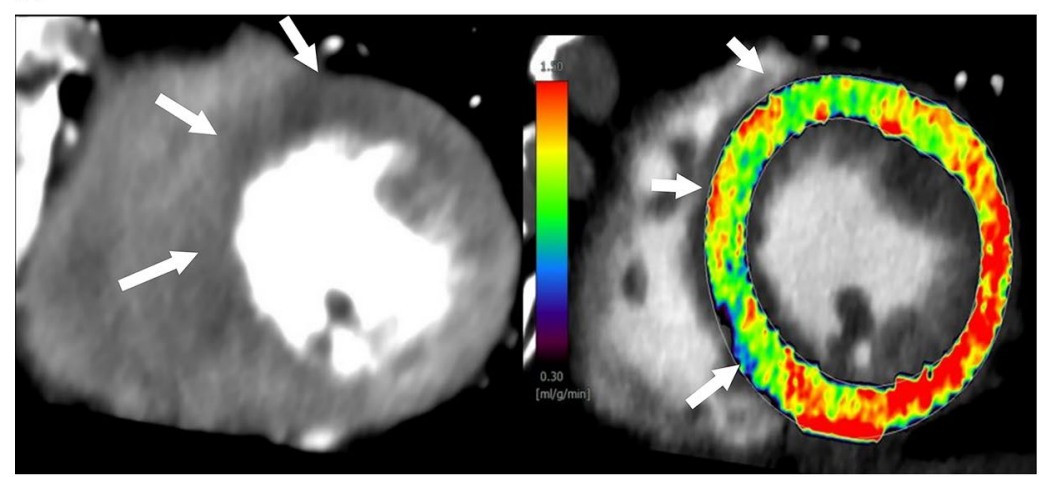

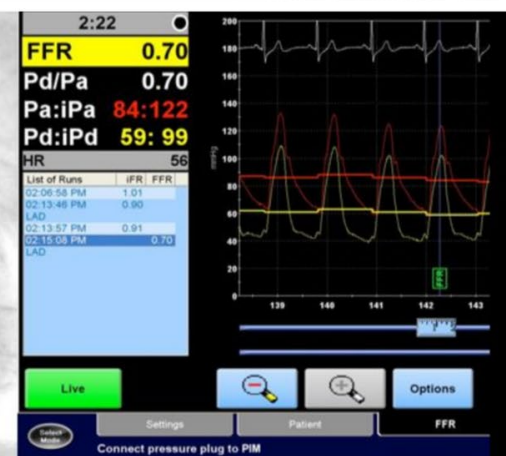

B

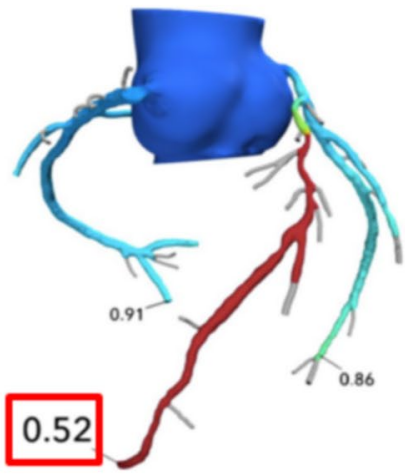

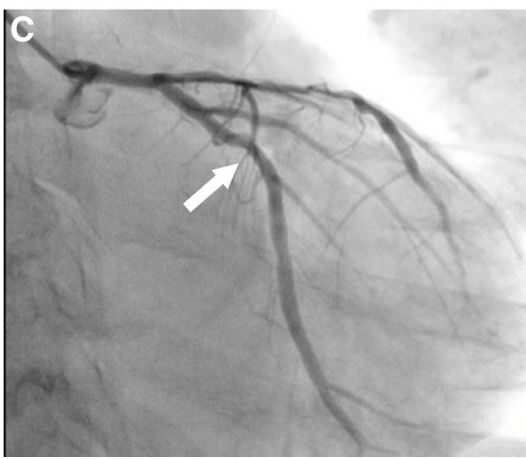

Fig. 13 CTP versus CT-FFR. a CTP image and CT-MBF map showing a perfusion defect and lower CT-MBF in the anteroseptal wall (arrows). b A CT-FFR image in the same patient showing abnormal CT-FFR with a low value of 0.52 at the LAD. c ICA showing a stenotic lesion in the mid LAD with an abnormal FFR of 0.70 .

better diagnostic performance than CTA alone [92]. Integrated CTP and CT-FFR has better performance than either of these techniques used alone [81].

\section{Conclusion}

Coronary CTA can exclude CAD with a high degree of certainty but has limited ability to evaluate the hemodynamic significance of stenosis because of its poor specificity. Emerging technologies such as CT-perfusion and CT-FFR can provide information on the hemodynamic significance of stenosis, which expands the capabilities of CT. These techniques make $\mathrm{CT}$ valuable for risk stratification and decisionmaking in patients with myocardial ischemia.

\section{Funding None.}

\section{Compliance with ethical standards}

Conflict of interest Yuki Tanabe declares that he has no conflict of interest. Akira Kurata declares that he has no conflict of interest. Takuya Matsuda declares that he has no conflict of interest. Kazuki Yoshida declares that he has no conflict of interest. Dhiraj Baruah is medical
$C T P$ computed tomography perfusion, $C T-M B F$ computed tomography derived-myocardial blood flow, $C T$ computed tomography, FFR fractional flow reserve, ICA invasive coronary angiography, $L A D$ left anterior descending artery

educator for Boehringer-Ingelheim pharmaceuticals, Inc. Teruhito Kido declares that he has no conflict of interest. Teruhito Mochizuki declares that he has no conflict of interest. Prabhakar Rajiah declares that he has no conflict of interest.

Ethical statement This article does not contain any studies with human participants or animals performed by any of the authors.

Open Access This article is licensed under a Creative Commons Attribution 4.0 International License, which permits use, sharing, adaptation, distribution and reproduction in any medium or format, as long as you give appropriate credit to the original author(s) and the source, provide a link to the Creative Commons licence, and indicate if changes were made. The images or other third party material in this article are included in the article's Creative Commons licence, unless indicated otherwise in a credit line to the material. If material is not included in the article's Creative Commons licence and your intended use is not permitted by statutory regulation or exceeds the permitted use, you will need to obtain permission directly from the copyright holder. To view a copy of this licence, visit http://creativecommons.org/licenses/by/4.0/.

\section{References}

1. Mori H, Torii S, Kutyna M, Sakamoto A, Finn AV, Virmani R, et al. Coronary artery calcification and its progression: what does it really mean? JACC Cardiovasc Imaging. 2018;11:127-42. 
2. Nesto RW, Kowalchuk GJ. The ischemic cascade: temporal sequence of hemodynamic, electrocardiocardiographic, and symptomatic expressions of ischemia. Am J Cardiol. 1987;59:23-30.

3. Stillman AE, Oudkerk M, Bluemke DA, de Boer MJ, Bremerich $\mathrm{J}$, Garcia EV, et al. Imaging the myocardial ischemic cascade. Int J Cardiovasc Imaging. 2018;34:1249-63.

4. Renker M, Baumann S, Rier J, Ebersberger U, Fuller SR, Batalis NI, et al. Imaging coronary artery disease and the myocardial ischemic cascade: clinical principles and scope. Radiol Clin N Am. 2015;53:261-9.

5. Tonino PA, De Bruyne B, Pijls NH, Siebert U, Ikeno F, van't Veer M, et al. Fractional flow reserve versus angiography for guiding percutaneous coronary intervention. N Engl J Med. 2009;360:213-24.

6. Hachamovitch R, Hayes SW, Friedman JD, Cohen I, Berman DS. Comparison of the short-term survival benefit associated with revascularization compared with medical therapy in patients with no prior coronary artery disease undergoing stress myocardial perfusion single photon emission computed tomography. Circulation. 2003;107:2900-7.

7. JCS 2018 Guideline on Diagnosis of Chronic Coronary Heart Diseases. https://www.j-circ.or.jp/guideline/pdf/JCS2018_yamag ishi_tamaki.pdf. Accessed 30 Dec 2019.

8. Wolk MJ, Bailey SR, Doherty JU, Douglas PS, Hendel RC, Kramer CM, et al. ACCF/AHA/ASE/ASNC/HFSA/HRS/SCAI/ SCCT/SCMR/STS 2013 multimodality appropriate use criteria for the detection and risk assessment of stable ischemic heart disease: a report of the American College of Cardiology Foundation Appropriate Use Criteria Task Force, American Heart Association, American Society of Echocardiography, American Society of Nuclear Cardiology, Heart Failure Society of America, Heart Rhythm Society, Society for Cardiovascular Angiography and Interventions, Society of Cardiovascular Computed Tomography, Society for Cardiovascular Magnetic Resonance, and Society of Thoracic Surgeons. J Am Coll Cardiol. 2014;63:380-406.

9. De Bruyne B, Pijls NH, Heyndrickx GR, Hodeige D, Kirkeeide $\mathrm{R}$, Gould KL. Pressure-derived fractional flow reserve to assess serial epicardial stenoses: theoretical basis and animal validation. Circulation. 2000;101:1840-7.

10. Curzen N, Rana O, Nicholas Z, Golledge P, Zaman A, Oldroyd $\mathrm{K}$, et al. Does routine pressure wire assessment influence management strategy at coronary angiography for diagnosis of chest pain?: the RIPCORD study. Circ Cardiovasc Interv. 2014;7:248-55.

11. Zimmermann FM, Ferrara A, Johnson NP, van Nunen LX, Escaned J, Albertsson P, et al. Deferral vs performance of percutaneous coronary intervention of functionally non-significant coronary stenosis: 15 year follow up of the DEFER trial. Eur Heart J. 2015;36:3182-8.

12. De Bruyne B, Pijls NH, Kalesan B, Barbato E, Tonino PA, Piroth Z, et al. Fractional flow reserve-guided PCI versus medical therapy in stable coronary artery disease. N Engl J Med. 2012;367:991-1001.

13. Pijls NH, De Bruyne B, Peels K, Van Der Voort PH, Bonnier $\mathrm{HJ}$, Bartunek J, et al. Measurement of fractional flow reserve to assess the functional severity of coronary-artery stenoses. N Engl J Med. 1996;334:1703-8.

14. The Organisation for Economic Co-operation and Development. OECD Health Statistics 2019. 2019. https://www.oecd.org/els/ health-systems/health-data.htm. Accessed 5 Jan 2020.

15. JROAD (The Japanese Registry of All cardiac and vascular Diseases). 2018. https://www.j-circ.or.jp/jittai_chosa/jittai_chosa 2017web.pdf. Accessed 5 Jan 2020.
16. Schroeder S, Achenbach S, Bengel F, Burgstahler C, Cademartiri F, de Feyter P, et al. Cardiac computed tomography: indications, applications, limitations, and training requirements: report of a Writing Group deployed by the Working Group Nuclear Cardiology and Cardiac CT of the European Society of Cardiology and the European Council of Nuclear Cardiology. Eur Heart J. 2008;29:531-56.

17. Patel MR, Peterson ED, Dai D, Brennan JM, Redberg RF, Anderson HV, et al. Low diagnostic yield of elective coronary angiography. N Engl J Med. 2010;362:886-95.

18. Meijboom WB, Van Mieghem CA, van Pelt N, Weustink A, Pugliese F, Mollet NR, et al. Comprehensive assessment of coronary artery stenoses: computed tomography coronary angiography versus conventional coronary angiography and correlation with fractional flow reserve in patients with stable angina. J Am Coll Cardiol. 2008;52:636-43.

19. Hecht H. CT derived FFR: "The game changer?" revisited. J Cardiovasc Comput Tomogr. 2018;12:447-9.

20. Cury RC, Abbara S, Achenbach S, Agatston A, Berman DS, Budoff MJ, et al. CAD-RADS ${ }^{\mathrm{TM}}$ Coronary Artery DiseaseReporting and Data System. An expert consensus document of the Society of Cardiovascular Computed Tomography (SCCT), the American College of Radiology (ACR) and the North American Society for Cardiovascular Imaging (NASCI). Endorsed by the American College of Cardiology. J Cardiovasc Comput Tomogr. 2016;10:269-81.

21. Rossi A, Merkus D, Klotz E, Mollet N, de Feyter PJ, Krestin GP. Stress myocardial perfusion: imaging with multidetector CT. Radiology. 2014;270:25-46.

22. Gould KL, Lipscomb K, Hamilton GW. Physiologic basis for assessing critical coronary stenosis. Instantaneous flow response and regional distribution during coronary hyperemia as measures of coronary flow reserve. Am J Cardiol. 1974;33:87-94.

23. Henzlova MJ, Duvall WL, Einstein AJ, Travin MI, Verberne HJ. ASNC imaging guidelines for SPECT nuclear cardiology procedures: stress, protocols, and tracers. J Nucl Cardiol. 2016;23:606-39.

24. Saab R, Hage FG. Vasodilator stress agents for myocardial perfusion imaging. J Nucl Cardiol. 2017;24:434-8.

25. Techasith T, Cury RC. Stress myocardial CT perfusion: an update and future perspective. JACC Cardiovasc Imaging. 2011;4:905-16.

26. Kurata A, Mochizuki T, Koyama Y, Haraikawa T, Suzuki J, Shigematsu Y, et al. Myocardial perfusion imaging using adenosine triphosphate stress multi-slice spiral computed tomography. Circ J. 2005;69:550-7.

27. Bischoff B, Bamberg F, Marcus R, Schwarz F, Becker HC, Becker A, et al. Optimal timing for first-pass stress CT myocardial perfusion imaging. Int J Cardiovasc Imaging. 2013;29:435-42.

28. Tanabe Y, Kido T, Kurata A, Uetani T, Fukuyama N, Yokoi T, et al. Optimal scan time for single-phase myocardial computed tomography perfusion to detect myocardial ischemia-derivation cohort from dynamic myocardial computed tomography perfusion. Circ J. 2016;80:2506-12.

29. Pontone G, Baggiano A, Andreini D, Guaricci AI, Guglielmo M, Muscogiuri G, et al. Diagnostic accuracy of simultaneous evaluation of coronary arteries and myocardial perfusion with single stress cardiac computed tomography acquisition compared to invasive coronary angiography plus invasive fractional flow reserve. Int J Cardiol. 2018;273:263-8.

30. Pontone G, Baggiano A, Andreini D, Guaricci AI, Guglielmo M, Muscogiuri G, et al. Dynamic stress computed tomography perfusion with a whole-heart coverage scanner in addition to coronary computed tomography angiography and fractional flow reserve computed tomography derived. JACC Cardiovasc 
Imaging. 2019. https://doi.org/10.1016/j.jcmg.2019.02.015(epub ahead of print).

31. Nagao M, Matsuoka H, Kawakami H, Higashino H, Mochizuki $\mathrm{T}$, Uemura $\mathrm{M}$, et al. Myocardial ischemia in acute coronary syndrome: assessment using 64-MDCT. AJR Am J Roentgenol. 2009;193:1097-106.

32. Leipsic J, Labounty TM, Hague CJ, Mancini GB, O'Brien JM, Wood DA, et al. Effect of a novel vendor-specific motion-correction algorithm on image quality and diagnostic accuracy in persons undergoing coronary $\mathrm{CT}$ angiography without rate-control medications. J Cardiovasc Comput Tomogr. 2012;6:164-71.

33. Dai X, Yu M, Pan J, Lu Z, Shen C, Wang Y, et al. Image quality and diagnostic accuracy of coronary CT angiography derived from low-dose dynamic CT myocardial perfusion: a feasibility study with comparison to invasive coronary angiography. Eur Radiol. 2018;29:4349-56.

34. Coenen A, Lubbers MM, Kurata A, Kono A, Dedic A, Chelu $\mathrm{RG}$, et al. Diagnostic value of transmural perfusion ratio derived from dynamic CT-based myocardial perfusion imaging for the detection of haemodynamically relevant coronary artery stenosis. Eur Radiol. 2017;27:2309-16.

35. Tanabe Y, Kido T, Uetani T, Kurata A, Kono T, Ogimoto A, et al. Differentiation of myocardial ischemia and infarction assessed by dynamic computed tomography perfusion imaging and comparison with cardiac magnetic resonance and single-photon emission computed tomography. Eur Radiol. 2016;26:3790-801.

36. Kikuchi Y, Oyama-Manabe N, Naya M, Manabe O, Tomiyama Y, Sasaki T, et al. Quantification of myocardial blood flow using dynamic 320-row multi-detector CT as compared with ${ }^{15} \mathrm{O}-\mathrm{H}_{2} \mathrm{O}$ PET. Eur Radiol. 2014;24:1547-56.

37. Danad I, Szymonifka J, Schulman-Marcus J, Min JK. Static and dynamic assessment of myocardial perfusion by computed tomography. Eur Heart J Cardiovasc Imaging. 2016;17:836-44.

38. So A, Imai Y, Nett B, Jackson J, Nett L, Hsieh J, et al. Technical note: evaluation of a $160-\mathrm{mm} / 256$-row CT scanner for wholeheart quantitative myocardial perfusion imaging. Med Phys. 2016;43:4821.

39. Tanabe Y, Kido T, Kurata A, Kouchi T, Hosokawa T, Nishiyama $\mathrm{H}$, et al. Impact of knowledge-based iterative model reconstruction on image quality and hemodynamic parameters in dynamic myocardial computed tomography perfusion using low-tubevoltage scan: a feasibility study. J Comput Assist Tomogr. 2019;43:811-6.

40. Tomizawa N, Chou S, Fujino Y, Kamitani M, Yamamoto K, Inoh $\mathrm{S}$, et al. Feasibility of dynamic myocardial CT perfusion using single-source 64-row CT. J Cardiovasc Comput Tomogr. 2019;13:55-61.

41. Ihdayhid AR, Sakaguchi T, Linde JJ, Sørgaard MH, Kofoed KF, Fujisawa Y, et al. Performance of computed tomography-derived fractional flow reserve using reduced-order modelling and static computed tomography stress myocardial perfusion imaging for detection of haemodynamically significant coronary stenosis. Eur Heart J Cardiovasc Imaging. 2018;19:1234-43.

42. Danad I, Fayad ZA, Willemink MJ, Min JK. New applications of cardiac computed tomography: dual-energy, spectral, and molecular CT imaging. JACC Cardiovasc Imaging. 2015;8:710-23.

43. Kalisz K, Halliburton S, Abbara S, Leipsic JA, Albrecht MH, Schoepf UJ, et al. Update on cardiovascular applications of multienergy CT. Radiographics. 2017;37:1955-74.

44. Delgado Sánchez-Gracián C, Oca Pernas R, Trinidad López C, Santos Armentia E, Vaamonde Liste A, Vázquez Caamaño $\mathrm{M}$, et al. Quantitative myocardial perfusion with stress dualenergy CT: iodine concentration differences between normal and ischemic or necrotic myocardium. Initial experience. Eur Radiol. 2016;26:3199-207.
45. Pelgrim GJ, van Hamersvelt RW, Willemink MJ, Schmidt BT, Flohr T, Schilham A, et al. Accuracy of iodine quantification using dual energy CT in latest generation dual source and dual layer CT. Eur Radiol. 2017;27:3904-12.

46. Carrascosa PM, Cury RC, Deviggiano A, Capunay C, Campisi R, López de Munain M, et al. Comparison of myocardial perfusion evaluation with single versus dual-energy CT and effect of beam-hardening artifacts. Acad Radiol. 2015;22:591-9.

47. Rodriguez-Granillo GA, Carrascosa P, Cipriano S, De Zan $\mathrm{M}$, Deviggiano A, Capunay C, et al. Beam hardening artifact reduction using dual energy computed tomography: implications for myocardial perfusion studies. Cardiovasc Diagn Ther. 2015;5:79-85.

48. Tanabe Y, Kido T, Kurata A, Kouchi T, Fukuyama N, Yokoi $\mathrm{T}$, et al. Late iodine enhancement computed tomography with image subtraction for assessment of myocardial infarction. Eur Radiol. 2018;28:1285-92.

49. Goetti R, Feuchtner G, Stolzmann P, Donati OF, Wieser M, Plass A, et al. Delayed enhancement imaging of myocardial viability: low-dose high-pitch CT versus MRI. Eur Radiol. 2011;21:2091-9.

50. Aikawa T, Oyama-Manabe N, Naya M, Ohira H, Sugimoto A, Tsujino I, et al. Delayed contrast-enhanced computed tomography in patients with known or suspected cardiac sarcoidosis: a feasibility study. Eur Radiol. 2017;27:4054-63.

51. Kurobe Y, Kitagawa K, Ito T, Kurita Y, Shiraishi Y, Nakamori $\mathrm{S}$, et al. Myocardial delayed enhancement with dual-source CT: advantages of targeted spatial frequency filtration and image averaging over half-scan reconstruction. J Cardiovasc Comput Tomogr. 2014;8:289-98.

52. Tanabe Y, Kido T, Kurata A, Fukuyama N, Yokoi T, Kido $\mathrm{T}$, et al. Impact of knowledge-based iterative model reconstruction on myocardial late iodine enhancement in computed tomography and comparison with cardiac magnetic resonance. Int J Cardiovasc Imaging. 2017;33:1609-18.

53. Ohta Y, Kitao S, Yunaga H, Fujii S, Mukai N, Yamamoto $\mathrm{K}$, et al. Myocardial delayed enhancement CT for the evaluation of heart failure: comparison to MRI. Radiology. 2018;288:682-91.

54. George RT, Mehra VC, Chen MY, Kitagawa K, Arbab-Zadeh A, Miller JM, et al. Myocardial CT perfusion imaging and SPECT for the diagnosis of coronary artery disease: a head-to-head comparison from the CORE320 multicenter diagnostic performance study. Radiology. 2014;272:407-16.

55. Tamarappoo BK, Dey D, Nakazato R, Shmilovich H, Smith T, Cheng VY, et al. Comparison of the extent and severity of myocardial perfusion defects measured by CT coronary angiography and SPECT myocardial perfusion imaging. JACC Cardiovasc Imaging. 2010;3:1010-9.

56. Cerqueira MD, Weissman NJ, Dilsizian V, Jacobs AK, Kaul S, Laskey WK, American Heart Association Writing Group on Myocardial Segmentation, and Registration for Cardiac Imaging, et al. (2002) Standardized myocardial segmentation and nomenclature for tomographic imaging of the heart. A statement for healthcare professionals from the Cardiac Imaging Committee of the Council on Clinical Cardiology of the American Heart Association. Circulation. 2002;105:539-42.

57. Kitagawa K, George RT, Arbab-Zadeh A, Lima JA, Lardo AC, et al. Characterization and correction of beam-hardening artifacts during dynamic volume $\mathrm{CT}$ assessment of myocardial perfusion. Radiology. 2010;256:111-8.

58. George RT, Arbab-Zadeh A, Miller JM, Kitagawa K, Chang HJ, Bluemke DA, et al. Adenosine stress 64- and 256-row detector computed tomography angiography and perfusion imaging: a pilot study evaluating the transmural extent of perfusion 
abnormalities to predict atherosclerosis causing myocardial ischemia. Circ Cardiovasc Imaging. 2009;2:174-82.

59. Pontone G, Andreini D, Guaricci AI, Guglielmo M, Baggiano A, Muscogiuri G, et al. Quantitative vs. qualitative evaluation of static stress computed tomography perfusion to detect haemodynamically significant coronary artery disease. Eur Heart $\mathbf{J}$ Cardiovasc Imaging. 2018;19:1244-52.

60. Nieman K, Shapiro MD, Ferencik M, Nomura CH, Abbara S, Hoffmann U, et al. Reperfused myocardial infarction: contrastenhanced 64-Section CT in comparison to MR imaging. Radiology. 2008;247:49-56.

61. Huber AM, Leber V, Gramer BM, Muenzel D, Leber A, Rieber $\mathrm{J}$, et al. Myocardium: dynamic versus single-shot CT perfusion imaging. Radiology. 2013;269:378-86.

62. Greif M, von Ziegler F, Bamberg F, Tittus J, Schwarz F, D'Anastasi M, et al. CT stress perfusion imaging for detection of haemodynamically relevant coronary stenosis as defined by FFR. Heart. 2013;99:1004-111.

63. van Assen M, Pelgrim GJ, De Cecco CN, Stijnen JMA, Zaki $\mathrm{BM}$, Oudkerk M, et al. Intermodel disagreement of myocardial blood flow estimation from dynamic CT perfusion imaging. Eur J Radiol. 2019;110:175-80.

64. Ishida M, Kitagawa K, Ichihara T, Natsume T, Nakayama R, Nagasawa N, et al. Underestimation of myocardial blood flow by dynamic perfusion CT: Explanations by two-compartment model analysis and limited temporal sampling of dynamic CT. J Cardiovasc Comput Tomogr. 2016;10:207-14.

65. Yang J, Dou G, He B, Jin Q, Chen Z, Jing J, et al. Stress myocardial blood flow ratio by dynamic $\mathrm{CT}$ perfusion identifies hemodynamically significant coronary artery disease. JACC Cardiovasc Imaging. 2019. https://doi.org/10.1016/j.jemg.2019.06.016(epub ahead of print).

66. Kuwahara N, Tanabe Y, Kido T, Kurata A, Uetani T, Ochi H, et al. Coronary artery stenosis-related perfusion ratio using dynamic computed tomography myocardial perfusion imaging: a pilot for identification of hemodynamically significant coronary artery disease. Cardiovasc Interv Ther. 2019. https://doi. org/10.1007/s12928-019-00627-4(epub ahead of print).

67. Liu A, Wijesurendra RS, Liu JM, Greiser A, Jerosch-Herold M, Forfar JC, Channon KM, et al. Gadolinium-free cardiac MR stress T1-mapping to distinguish epicardial from microvascular coronary disease. J Am Coll Cardiol. 2018;71:957-68.

68. Obara M, Naya M, Oyama-Manabe N, Aikawa T, Tomiyama Y, Sasaki T, et al. Diagnostic value of quantitative coronary flow reserve and myocardial blood flow estimated by dynamic 320 MDCT scanning in patients with obstructive coronary artery disease. Medicine (Baltimore). 2018;97:e11354.

69. Danad I, Uusitalo V, Kero T, Saraste A, Raijmakers PG, Lammertsma AA, et al. Quantitative assessment of myocardial perfusion in the detection of significant coronary artery disease: cutoff values and diagnostic accuracy of quantitative [(15)O]H2O PET imaging. J Am Coll Cardiol. 2014;64:1464-75.

70. Nakamori S, Sakuma H, Dohi K, Ishida M, Tanigawa T, Yamada A, et al. Combined assessment of stress myocardial perfusion cardiovascular magnetic resonance and flow measurement in the coronary sinus improves prediction of functionally significant coronary stenosis determined by fractional flow reserve in multivessel disease. J Am Heart Assoc. 2018;7(3):e007736.

71. van de Hoef TP, van Lavieren MA, Damman P, Delewi R, Piek MA, Chamuleau SA, et al. Physiological basis and long-term clinical outcome of discordance between fractional flow reserve and coronary flow velocity reserve in coronary stenoses of intermediate severity. Circ Cardiovasc Interv. 2014;7:301-11.

72. Tang K, Wang L, Li R, Lin J, Zheng X, Cao G. Effect of low tube voltage on image quality, radiation dose, and low-contrast detectability at abdominal multidetector CT: phantom study. J Biomed Biotechnol. 2012;2012:130169.

73. Oda S, Utsunomiya D, Funama Y, Awai K, Katahira K, Nakaura $\mathrm{T}$, et al. A low tube voltage technique reduces the radiation dose at retrospective ECG-gated cardiac computed tomography for anatomical and functional analyses. Acad Radiol. 2011;18:991-9.

74. Yuki H, Utsunomiya D, Funama Y, Tokuyasu S, Namimoto T, Hirai T, et al. Value of knowledge-based iterative model reconstruction in low-kV 256-slice coronary CT angiography. J Cardiovasc Comput Tomogr. 2014;8:115-23.

75. Wu D, Kim K, El Fakhri G, Li Q. Iterative low-dose CT reconstruction with priors trained by artificial neural network. IEEE Trans Med Imaging. 2017;36:2479-86.

76. Wang Y, Liao Y, Zhang Y, He J, Li S, Bian Z, et al. Iterative quality enhancement via residual-artifact learning networks for low-dose CT. Phys Med Biol. 2018;63:215004.

77. Tatsugami F, Higaki T, Nakamura Y, Yu Z, Zhou J, Lu Y, et al. Deep learning-based image restoration algorithm for coronary CT angiography. Eur Radiol. 2019;29:5322-9.

78. Bettencourt N, Chiribiri A, Schuster A, Ferreira N, Sampaio F, Pires-Morais G, et al. Direct comparison of cardiac magnetic resonance and multidetector computed tomography stress-rest perfusion imaging for detection of coronary artery disease. J Am Coll Cardiol. 2013;61:1099-107.

79. Pontone G, Andreini D, Guaricci AI, Baggiano A, Fazzari F, Guglielmo M, et al. Incremental diagnostic value of stress computed tomography myocardial perfusion with whole-heart coverage CT scanner in intermediate- to high-risk symptomatic patients suspected of coronary artery disease. JACC Cardiovasc Imaging. 2019;12:338-49.

80. Shikata F, Imagawa H, Kawachi K, Kido T, Kurata A, Inoue Y, et al. Regional myocardial blood flow measured by stress multidetector computed tomography as a predictor of recovery of left ventricular function after coronary artery bypass grafting. Am Heart J. 2010;160:528-34.

81. Coenen A, Rossi A, Lubbers MM, Kurata A, Kono AK, Chelu $\mathrm{RG}$, et al. Integrating CT myocardial perfusion and CT-FFR in the work-up of coronary artery disease. JACC Cardiovasc Imaging. 2017;10:760-70.

82. Minhas A, Dewey M, Vavere AL, Tanami Y, Ostovaneh MR, Laule M, et al. Patient preferences for coronary CT angiography with stress perfusion, SPECT, or invasive coronary angiography. Radiology. 2019;291:340-8.

83. Meyer M, Nance JW Jr, Schoepf UJ, Moscariello A, Weininger $\mathrm{M}$, Rowe GW, et al. Cost-effectiveness of substituting dualenergy CT for SPECT in the assessment of myocardial perfusion for the workup of coronary artery disease. Eur J Radiol. 2012;81:3719-25.

84. Hamon M, Geindreau D, Guittet L, Bauters C, Hamon M. Additional diagnostic value of new CT imaging techniques for the functional assessment of coronary artery disease: a meta-analysis. Eur Radiol. 2019;29:3044-61.

85. Takx RA, Blomberg BA, El Aidi H, Habets J, de Jong PA, Nagel E, et al. Diagnostic accuracy of stress myocardial perfusion imaging compared to invasive coronary angiography with fractional flow reserve meta-analysis. Circ Cardiovasc Imaging. 2015. https://doi.org/10.1161/CIRCIMAGING.114.002666.

86. Yang DH, Kim YH, Roh JH, Kang JW, Han D, Jung J, et al. Stress myocardial perfusion CT in patients suspected of having coronary artery disease: visual and quantitative analysis-validation by using fractional flow reserve. Radiology. 2015;276:715-23.

87. Yang DH, Kim YH, Roh JH, Kang JW, Ahn JM, Kweon J, et al. Diagnostic performance of on-site CT-derived fractional flow reserve versus CT perfusion. Eur Heart J Cardiovasc Imaging. 2017;18:432-40. 
88. Rossi A, Dharampal A, Wragg A, Davies LC, van Geuns RJ, Anagnostopoulos C, et al. Diagnostic performance of hyperaemic myocardial blood flow index obtained by dynamic computed tomography: does it predict functionally significant coronary lesions? Eur Heart J Cardiovasc Imaging. 2014;15:85-94.

89. Lu M, Wang S, Sirajuddin A, Arai AE, Zhao S. Dynamic stress computed tomography myocardial perfusion for detecting myocardial ischemia: a systematic review and meta-analysis. Int $\mathbf{J}$ Cardiol. 2018;258:325-31.

90. Celeng C, Leiner T, Maurovich-Horvat P, Merkely B, de Jong $\mathrm{P}$, Dankbaar JW, et al. Anatomical and functional computed tomography for diagnosing hemodynamically significant coronary artery disease: a meta-analysis. JACC Cardiovasc Imaging. 2019;12:1316-25.

91. Rochitte CE, George RT, Chen MY, Arbab-Zadeh A, Dewey M, Miller JM, et al. Computed tomography angiography and perfusion to assess coronary artery stenosis causing perfusion defects by single photon emission computed tomography: the CORE320 study. Eur Heart J. 2014;35:1120-30.

92. Pontone G, Baggiano A, Andreini D, Guaricci AI, Guglielmo M, Muscogiuri G, et al. Stress computed tomography perfusion versus fractional flow reserve CT derived in suspected coronary artery disease. JACC Cardiovasc Imaging. 2019;12:1487-97.

93. Lubbers M, Coenen A, Kofflard M, Bruning T, Kietselaer B, Galema T, et al. Comprehensive cardiac CT with myocardial perfusion imaging versus functional testing in suspected coronary artery disease: the multicenter, randomized CRESCENT-II trial. JACC Cardiovasc Imaging. 2018;11:1625-36.

94. Chen MY, Rochitte CE, Arbab-Zadeh A, Dewey M, George RT, Miller JM, et al. Prognostic value of combined CT angiography and myocardial perfusion imaging versus invasive coronary angiography and nuclear stress perfusion imaging in the prediction of major adverse cardiovascular events: the CORE320 multicenter study. Radiology. 2017;284:55-65.

95. Nakamura S, Kitagawa K, Goto Y, Omori T, Kurita T, Yamada A, et al. Incremental prognostic value of myocardial blood flow quantified with stress dynamic computed tomography perfusion imaging. JACC Cardiovasc Imaging. 2019;12:1379-87.

96. van Assen M, De Cecco CN, Eid M, von Knebel DP, Scarabello M, Lavra F, et al. Prognostic value of CT myocardial perfusion imaging and CT-derived fractional flow reserve for major adverse cardiac events in patients with coronary artery disease. J Cardiovasc Comput Tomogr. 2019;13:26-33.

97. Taylor CA, Fonte TA, Min JK. Computational fluid dynamics applied to cardiac computed tomography for noninvasive quantification of fractional flow reserve: scientific basis. J Am Coll Cardiol. 2013;61:2233-41.

98. Koo BK, Erglis A, Doh JH, Daniels DV, Jegere S, Kim HS, et al. Diagnosis of ischemia causing coronary stenoses by noninvasive fractional flow reserve computed from coronary computed tomographic angiograms. Results from the prospective multicenter DISCOVER-FLOW (Diagnosis of Ischemia-Causing Stenoses Obtained Via Noninvasive Fractional Flow Reserve) study. J Am Coll Cardiol. 2011;58:1989-97.

99. Min JK, Leipsic J, Pencina MJ, Berman DS, Koo BK, van Mieghem C, et al. Diagnostic accuracy of fractional flow reserve from anatomic CT angiography. JAMA. 2012;308:1237-45.

100. Nørgaard BL, Leipsic J, Gaur S, Seneviratne S, Ko BS, Ito H, et al. Diagnostic performance of noninvasive fractional flow reserve derived from coronary computed tomography angiography in suspected coronary artery disease: the NXT trial (Analysis of Coronary Blood Flow Using CT Angiography: Next Steps). J Am Coll Cardiol. 2014;63:1145-55.

101. Rabbat MG, Berman DS, Kern M, Raff G, Chinnaiyan K, Koweek L, et al. Interpreting results of coronary computed tomography angiography-derived fractional flow reserve in clinical practice. J Cardiovasc Comput Tomogr. 2017;11:383-8.

102. Itu L, Rapaka S, Passerini T, Georgescu B, Schwemmer C, Schoebinger $\mathrm{M}$, et al. A machine-learning approach for computation of fractional flow reserve from coronary computed tomography. J Appl Physiol. 1985;2016(121):42-52.

103. Coenen A, Kim YH, Kruk M, Tesche C, De Geer J, Kurata A, et al. Diagnostic accuracy of a machine-learning approach to coronary computed tomographic angiography-based fractional flow reserve: result from the MACHINE consortium. Circ Cardiovasc Imaging. 2018;11:e007217. https://doi.org/10.1161/ CIRCIMAGING.117.007217.

104. Hirohata K, Kano A, Goryu A, Ooga J, Hongo T, Higashi S, et al. A novel CT-FFR method for the coronary artery based on 4D-CT image analysis and structural fluid analysis. SPIE Med Imaging. 2015;26:9412-94.

105. Fujimoto S, Kawasaki T, Kumamaru KK, Kawaguchi Y, Dohi $\mathrm{T}$, Okonogi $\mathrm{T}$, et al. Diagnostic performance of on-site computed CT-fractional flow reserve based on fluid structure interactions: comparison with invasive fractional flow reserve and instantaneous wave-free ratio. Eur Heart J Cardiovasc Imaging. 2019;20:343-52.

106. Donnelly PM, Kolossváry M, Karády J, Ball PA, Kelly S, Fitzsimons D, et al. Experience with an on-site coronary computed tomography-derived fractional flow reserve algorithm for the assessment of intermediate coronary stenoses. Am J Cardiol. 2018;121:9-13.

107. van Hamersvelt RW, Michiel Voskuil M, de Jong PA, Willemink MJ, Išgum I, Leiner T. Diagnostic performance of on-site coronary CT angiography-derived fractional flow reserve based on patient-specific lumped parameter models. Radiol Cardiothorac Imaging. 2019;1:e190036.

108. De Bruyne B, Hersbach F, Pijls NH, Bartunek J, Bech JW, Heyndrickx GR, et al. Abnormal epicardial coronary resistance in patients with diffuse atherosclerosis but "normal" coronary angiography. Circulation. 2001;104:2401-6.

109. Fairbairn TA, Nieman K, Akasaka T, Nørgaard BL, Berman DS, Raff G, et al. Real-world clinical utility and impact on clinical decision-making of coronary computed tomography angiography-derived fractional flow reserve: lessons from the ADVANCE Registry. Eur Heart J. 2018;39:3701-11.

110. Rajiah P, Maroules CD. Myocardial ischemia testing with computed tomography: emerging strategies. Cardivasc Diagn Ther. 2017;7:475-88.

111. Modi BN, Sankaran S, Kim HJ, Ellis H, Rogers C, Taylor $\mathrm{CA}$, et al. Predicting physiological effect of revascularization in serially diseased coronary arteries. Circ Cardiovasc Interv. 2019;12:e007577.

112. Nørgaard BL, Hjort J, Gaur S, Hansson N, Bøtker HE, Leipsic J, et al. Clinical use of coronary CTA-derived FFR for decision making in stable CAD. JACC Cardiovasc Imaging. 2017; 10:541-50.

113. Renker M, Schoepf UJ, Wang R, Meinel FG, Rier JD, Bayer RR 2nd, et al. Comparison of diagnostic value of a novel noninvasive coronary computed tomography angiography method versus standard coronary angiography for assessing fractional flow reserve. Am J Cardiol. 2014;114:1303-8.

114. Sand NPR, Veien KT, Nielsen SS, Nørgaard BL, Larsen P, Johansen A, et al. Prospective comparison of FFR derived from coronary CT angiography with SPECT perfusion imaging in stable coronary artery disease: the ReASSESS study. JACC Cardiovasc Imaging. 2018;11:1640-50.

115. Driessen RS, Danad I, Stuijfzand WJ, Raijmakers PG, Schumacher SP, van Diemen PA, et al. Comparison of coronary computed tomography angiography, fractional flow reserve, and 
perfusion imaging for ischemia diagnosis. J Am Coll Cardiol. 2019;73:161-73.

116. De Geer J, Sandstedt M, Björkholm A, Alfredsson J, Janzon M, Engvall J, et al. Software based on-site estimation of fractional flow reserve using standard coronary $\mathrm{CT}$ angiography data. Acta Radiol. 2016;57:1186-92.

117. Kim KH, Doh JH, Koo BK, Min JK, Erglis A, Yang HM, et al. A novel noninvasive technology for treatment planning using virtual coronary stenting and computed tomography-derived computed fractional flow reserve. JACC Cardiovasc Interv. 2014;7:72-8.

118. Wardziak Ł, Kruk M, Pleban W, Demkow M, Rużyłło W, Dzielińska Z, et al. Coronary CTA enhanced with CTA basedFFR analysis provides higher diagnostic value than invasive coronary angiography in patients with intermediate coronary stenosis. J Cardiovasc Comput Tomogr. 2019;13:62-7.

119. Kurata A, Fukuyama N, Hirai K, Kawaguchi N, Tanabe Y, Okayama $\mathrm{H}$, et al. On-site computed tomography-derived fractional flow reserve using a machine-learning algorithm-clinical effectiveness in a retrospective multicenter cohort. Circ J. 2019;83:1563-71.

120. Baumann S, Renker M, Hetjens S, Fuller SR, Becher T, Loßnitzer D, et al. Comparison of coronary computed tomography angiography-derived vs invasive fractional flow reserve assessment: meta-analysis with subgroup evaluation of intermediate stenosis. Acad Radiol. 2016;23:1402-11.

121. Wu W, Pan DR, Foin N, Pang S, Ye P, Holm N, et al. Noninvasive fractional flow reserve derived from coronary computed tomography angiography for identification of ischemic lesions: a systematic review and meta-analysis. Sci Rep. 2016;6:29409.

122. Danad I, Szymonifka J, Twisk JWR, Norgaard BL, Zarins CK, Knaapen P, et al. Diagnostic performance of cardiac imaging methods to diagnose ischaemia-causing coronary artery disease when directly compared with fractional flow reserve as a reference standard: a meta-analysis. Eur Heart J. 2017;38:991-8.

123. Douglas PS, Pontone G, Hlatky MA, Patel MR, Norgaard BL, Byrne RA, et al. Clinical outcomes of fractional flow reserve by computed tomographic angiography-guided diagnostic strategies vs. usual care in patients with suspected coronary artery disease: the prospective longitudinal trial of FFR(CT): outcome and resource impacts study. Eur Heart J. 2015;36:3359-67.

124. Douglas PS, De Bruyne B, Pontone G, Patel MR, Norgaard $\mathrm{BL}$, Byrne RA, et al. 1-year outcomes of $\mathrm{FFR}_{\mathrm{CT}}$-guided care in patients with suspected coronary disease: the PLATFORM study. J Am Coll Cardiol. 2016;68:435-45.

125. Curzen NP, Nolan J, Zaman AG, Nørgaard BL, Rajani R. Does the routine availability of CT-derived FFR influence management of patients with stable chest pain compared to CT angiography alone?: the FFCTRIPCORD study. JACC Cardiovasc Imaging. 2016;9:1188-94.

126. Collet C, Onuma Y, Andreini D, Sonck J, Pompilio G, Mushtaq $\mathrm{S}$, et al. Coronary computed tomography angiography for heart team decision-making in multivessel coronary artery disease. Eur Heart J. 2018;39:3689-98.

127. Andreini D, Modolo R, Katagiri Y, Mushtaq S, Sonck J, Collet $\mathrm{C}$, et al. Impact of fractional flow reserve derived from coronary computed tomography angiography on heart team treatment decision-making in patients with multivessel coronary artery disease: insights from the SYNTAX III REVOLUTION trial. Circ Cardiovasc Interv. 2019;12:e007607. https://doi. org/10.1161/CIRCINTERVENTIONS.118.007607.

128. Hlatky MA, Saxena A, Koo BK, Erglis A, Zarins CK, Min JK. Projected costs and consequences of computed tomography-determined fractional flow reserve. Clin Cardiol. 2013;36:743-8.
129. Patel MR, Nørgaard BL, Fairbairn TA, Nieman K, Akasaka T, Berman DS, et al. 1-year impact on medical practice and clinical outcomes of FFR CT: the ADVANCE registry. JACC Cardiovasc Imaging. 2019. https://doi.org/10.1016/j. jcmg.2019.03.003(epub ahead of print).

130. Nørgaard BL, Leipsic J, Koo BK, Zarins CK, Jensen JM, Sand $\mathrm{NP}$, et al. Coronary computed tomography angiography derived fractional flow reserve and plaque stress. Curr Cardiovasc Imaging Rep. 2016;9:2.

131. Choi G, Lee JM, Kim HJ, Park JB, Sankaran S, Otake H, et al. Coronary artery axial plaque stress and its relationship with lesion geometry: applications of computational fluid dynamics to coronary CT angiography. JACC Cardiovasc Imaging. 2015;8:1156-66.

132. Park J, Lee JM, Koo BK, Choi G, Hwang D, Rhee TM, et al. Relevance of anatomical, plaque, and hemodynamic characteristics of non-obstructive coronary lesions in the prediction of risk for acute coronary syndrome. Eur Radiol. 2019;29:6119-288.

133. Abbara S, Blanke P, Maroules CD, Cheezum M, Choi AD, Han $\mathrm{BK}$, et al. SCCT guidelines for the performance and acquisition of coronary computed tomographic angiography: a report of the society of Cardiovascular Computed Tomography Guidelines Committee: endorsed by the North American Society for Cardiovascular Imaging (NASCI). J Cardioasc Comput Tomogr. 2016;10:435-49.

134. Leipsic J, Yang TH, Thompson A, Koo BK, Mancini GB, Taylor C, et al. CT angiography (CTA) and diagnostic performance of noninvasive fractional flow reserve by anatomic CTA (DeFACTO) study. AJR Am J Roentgenol. 2014;202:989-94.

135. Budoff MJ, Dowe D, Jollis JG, Gitter M, Sutherland J, Halamert E, et al. Diagnostic performance of 64-multidetector row coronary computed tomographic angiography for evaluation of coronary artery stenosis in individuals without known coronary artery disease: results from the prospective multicenter ACCURACY (Assessment by Coronary Computed Tomographic Angiography of Individuals Undergoing Invasive Coronary Angiography) trial. J Am Coll Cardiol. 2008;52:1724-32.

136. Tesche C, Otani K, De Cecco CN, De Geer J, Kruk M, et al. Influence of coronary calcium on diagnostic performance of machine learning CT-FFR: results from MACHINE Registry. JACC Cardiovasc Imaging. 2019. https://doi.org/10.1016/j. jcmg.2019.06.027(epub ahead of print).

137. Pontone G, Weir-McCall JR, Baggiano A, Del Torto A, Fusini L, Guglielmo M, et al. Determinants of rejection rate for coronary CT angiography fractional flow reserve analysis. Radiology. 2019;292:597-605.

138. Gaur S, Bezerra HG, Lassen JF, Christiansen EH, Tanaka K, Jensen JM, et al. Fractional flow reserve derived from coronary CT angiography: variation of repeated analysis. J Cardiovasc Comput Tomogr. 2014;8:307-14.

139. Cook CM, Petraco R, Shun-Shin MJ, Ahmad Y, Nijjer S, AlLamee R, et al. Diagnostic accuracy of computed tomographyderived fractional flow reserve: a systematic review. JAMA Cardiol. 2017;2:803-10.

140. Narula J, Nakano M, Virmani R, Kolodgie FD, Petersen R, Newcomb R, et al. Histopathologic characteristics of atherosclerotic coronary disease and implications of the findings for the invasive and noninvasive detection of vulnerable plaques. J Am Coll Cardiol. 2013;61:1041-51.

141. Ahmadi A, Stone GW, Leipsic J, Serruys PW, Shaw L, Hecht $\mathrm{H}$, et al. Association of coronary stenosis and plaque morphology with fractional flow reserve and outcomes. JAMA Cardiol. 2016;1:350-7. https://doi.org/10.1001/jamacardio.2016.0263. 
142. Shaw LJ, Nicol E. Lesion-specific ischemia with noninvasive computed tomographic angiography: superior to conventional stress testing? JAMA Cardiol. 2017;2:717-9.

143. Arbab-Zadeh A, Miller JM, Rochitte CE, Dewey M, Niinuma $\mathrm{H}$, Gottlieb I, et al. Diagnostic accuracy of computed tomography coronary angiography according to pre-test probability of coronary artery disease and severity of coronary arterial calcification. The CORE-64 (Coronary Artery Evaluation Using 64-Row Multidetector Computed Tomography Angiography) International Multicenter Study. J Am Coll Cardiol. 2012;59:379-87.

144. Sharma RK, Arbab-Zadeh A, Kishi S, Chen MY, Magalhães TA, George RT, et al. Incremental diagnostic accuracy of computed tomography myocardial perfusion imaging over coronary angiography stratified by pre-test probability of coronary artery disease and severity of coronary artery calcification: the CORE320 study. Int J Cardiol. 2015;201:570-7.
145. Gonzalez JA, Lipinski MJ, Flors L, Shaw PW, Kramer CM, Salerno M, et al. Meta-analysis of diagnostic performance of coronary computed tomography angiography, computed tomography perfusion, and computed tomography-fractional flow reserve in functional myocardial ischemia assessment versus invasive fractional flow reserve. Am J Cardiol. 2015;116:1469-78.

146. Li Y, Yu M, Dai X, Lu Z, Shen C, Wang Y, et al. Detection of hemodynamically significant coronary stenosis: CT myocardial perfusion versus machine learning CT fractional flow reserve. Radiology. 2019;293:305-14.

Publisher's Note Springer Nature remains neutral with regard to jurisdictional claims in published maps and institutional affiliations. 